\title{
Genomic analysis of the domestication and post-Spanish conquest evolution of the llama and alpaca
}

Ruiwen $\mathrm{Fan}^{1 \dagger}{ }^{\dagger}$, Zhongru Gu${ }^{2,3,4+}$, Xuanmin Guang ${ }^{5 \dagger}$, Juan Carlos Marín ${ }^{6 \dagger}$, Valeria Varas ${ }^{7}$, Benito A. González ${ }^{8}$, Jane C. Wheeler ${ }^{9}$, Yafei Hư ${ }^{5}$, Erli Li ${ }^{5}$, Xiaohui Sun ${ }^{5}$, Xukui Yang ${ }^{5}$, Chi Zhang ${ }^{5}$, Wenjun Gao', Junping He', Kasper Munch ${ }^{10}$, Russel Corbett-Detig ${ }^{11}$, Mario Barbato ${ }^{12}$, Shengkai Pan ${ }^{2,3}$, Xiangjiang Zhan ${ }^{2,3,13^{*}+}$, Michael W. Bruford ${ }^{3,14^{*}+}$ and Changsheng Dong ${ }^{1 *+}$

\author{
* Correspondence: zhanxj@ioz.ac.cn; \\ BrufordMW@cardiff.ac.uk; \\ csdong18@163.com \\ ${ }^{\dagger}$ Ruiwen Fan, Zhongru Gu, Xuanmin \\ Guang, and Juan Carlos Marín are \\ joint first authors. \\ ${ }^{+}$Xiangjiang Zhan, Michael W \\ Bruford, and Changsheng Dong are \\ joint senior authors. \\ ${ }^{2}$ CAS Key Lab of Animal Ecology \\ and Conservation Biology, Institute \\ of Zoology, Chinese Academy of \\ Sciences, Beijing, China \\ ${ }^{3}$ Cardiff University - Institute of \\ Zoology Joint Laboratory for \\ Biocomplexity Research, Chinese \\ Academy of Sciences, Beijing, China \\ ${ }^{1}$ College of Animal Science and \\ Veterinary Medicine, Shanxi \\ Agricultural University, Taigu, \\ Shanxi, China \\ Full list of author information is \\ available at the end of the article
}

\begin{abstract}
Background: Despite their regional economic importance and being increasingly reared globally, the origins and evolution of the llama and alpaca remain poorly understood. Here we report reference genomes for the llama, and for the guanaco and vicuña (their putative wild progenitors), compare these with the published alpaca genome, and resequence seven individuals of all four species to better understand domestication and introgression between the llama and alpaca.

Results: Phylogenomic analysis confirms that the llama was domesticated from the guanaco and the alpaca from the vicuña. Introgression was much higher in the alpaca genome (36\%) than the llama (5\%) and could be dated close to the time of the Spanish conquest, approximately 500 years ago. Introgression patterns are at their most variable on the $X$-chromosome of the alpaca, featuring 53 genes known to have deleterious $X$-linked phenotypes in humans. Strong genome-wide introgression signatures include olfactory receptor complexes into both species, hypertension resistance into alpaca, and fleece/fiber traits into llama. Genomic signatures of domestication in the llama include male reproductive traits, while in alpaca feature fleece characteristics, olfaction-related and hypoxia adaptation traits. Expression analysis of the introgressed region that is syntenic to human HSA4q21, a gene cluster previously associated with hypertension in humans under hypoxic conditions, shows a previously undocumented role for PRDM8 downregulation as a potential transcriptional regulation mechanism, analogous to that previously reported at high altitude for hypoxia-inducible factor 1 a.
\end{abstract}

Conclusions: The unprecedented introgression signatures within both domestic camelid genomes may reflect post-conquest changes in agriculture and the breakdown of traditional management practices.

Keywords: Introgression, Domestication, Llama, Alpaca, Spanish conquest 


\section{Background}

South and Central America encompasses one of the most important cradles of domestication, giving rise to key crop plants and domesticated animals including turkey, guinea pig, and the region's key domestic herbivores, the llama and alpaca [1]. These South American camelids (SACs) attained central importance in the economy of the Andean region, with the llama used as a pack animal, facilitating the expansion of the Inca Empire [2], and the alpaca selected to produce some of the world's finest wool [3]. Both putative wild ancestors (the guanaco and vicuña) remain extant, with SACs classified in two genera, each containing two species: Lama guanicoe (guanaco) and L. glama (llama), and Vicugna vicugna (vicuña) and $V$. pacos (alpaca) [4, 5]. The alpaca has been variously hypothesized as descending from the guanaco, the vicuña, and as a llama/vicuña hybrid, while the llama is generally thought to originate from the guanaco [4]. These hypotheses stem from studies of morphological and behavioral variations in extant animals, while archaeozoological evidence places domestication of the alpaca from the vicuña in the wet puna ecosystem of Peru's central Andes, 6000-7000 years ago, and domestication of the llama from the guanaco in the dry punas of southern Peru, Chile, and Argentina [6]. Previous molecular data, while confirming that the genera Lama and Vicugna are valid and separated 2-3 MYA (million years ago), have documented extensive hybridization among modern domestic SACs, with microsatellite and mitochondrial DNA analysis suggesting that the llama is descended from the guanaco and the alpaca is descended from the vicuña, and the alpaca has been reclassified from Lama pacos to Vicuna pacos [4, 5]. Recent Y-chromosome analysis has recapitulated this result [7]. A genomic perspective on this problem is urgently needed, however, because previous analysis used a few neutral genetic markers, representing only a very small fraction of the genome, and was incapable of accurately assessing genetic diversity, genomic regions under selection and systematically evaluating genomewide introgression in wild and domestic forms.

We generated de novo genomes and transcriptomes for vicuña, llama, and guanaco, adding to a reference genome previously produced for alpaca [8] and resequenced the genomes of seven further individuals of each species. We aimed to elucidate the following for the llama and alpaca: (1) their evolutionary origins; (2) the level, origin, distribution of introgression in both species; (3) their demographic history from domestication to the present day, including the Spanish conquest; and (4) genes selected during domestication and potentially as a result of admixture, and their implication for the llama and alpaca's unique adaptations.

\section{Results}

De novo sequencing, assembly, and resequencing

Genomes were sequenced to 129x, 108x, and 102x coverage for L. guanicoe, V. vicugna, and L. glama, respectively (Additional file 1: Supplementary Text 1a, b and Additional file 1: Table S1); all these genomes were assembled by SOAPdenovo [9] and featuring contig and scaffold N50 lengths of $91.6 \mathrm{~kb}$ and $14.6 \mathrm{Mb}$ for L. guanicoe, $91.1 \mathrm{~kb}$ and $6.15 \mathrm{Mb}$ for $V$. vicugna, and $44.1 \mathrm{~kb}$ and $3.2 \mathrm{Mb}$ for L. glama (Additional file 1: Table S2ac). Each species' blood was used for RNA sequencing and those assembled unigene alignment showed that the assemblies covered more than $82 \%$ of unique genes for each species (Additional file 1: Table S3). Benchmarking Universal Single-Copy Orthologs (BUSCO) 
assessment showed that $>93.7 \%$ of core genes [10] could be aligned to the SAC genomes (Additional file 1: Table S4). The three de novo genomes were all inferred to be $\sim 2.6 \mathrm{~Gb}$ (Additional file 1: Table S5 and Figure S1) and share high synteny with the human and $V$. pacos reference genomes (coverage rate $87 \%$; Additional file 1: Table S6), with a relatively low rate of genomic rearrangement. We identified 21,435 protein-coding genes in the genomes of L. guanicoe, 21,757 in V. vicugna, and 21,460 in L. glama, respectively (Additional file 1: Table S7a-c). BUSCO assessment showed that the large majority present in the predicted gene sets (over $78 \%$ ). In total, $>92 \%$ of genes was functionally annotated. Repeat sequences were inferred to comprised $33.1 \%$ of the genome in L. guanicoe, 32.7\% in V. vicugna, and $27.02 \%$ in L. glama, lower values than in most mammals [11], although such sequences were difficult to assemble most likely due to the limitations of short-read sequencing. Resequencing of seven individuals per species (Additional file 1: Figure S2; Table S8) yielded an average sequence depth of 16-fold after filtering (31-45 Gb, Additional file 1: Table S9). Resequencing uncovered 21.3 million SNPs and a heterozygous SNP rate of 0.0030 for alpaca, 25.2 million and 0.0023 for vicuña, 16.7 million and 0.0018 for guanaco, and 16.1 million and 0.0020 for llama, indicating a higher overall SNP rate for the Vicugna lineage.

\section{Phylogenetic, admixture, and lineage sorting analysis}

A neighbor-joining tree based on 5,901,447 SNPs (Fig. 1a upper) showed two major SAC lineages with 100\% bootstrap support, comprising vicuña and alpaca in one group (genus Vicugna) and guanaco and llama in the other (genus Lama). Within Lama, L. g. guanicoe formed a single group, whereas two L. g. cacsilensis (guanaco 22 and 24) grouped with L. g. guanicoe, the other two (guanaco138DA and 8) grouped with all llamas. Guanaco 22 and 24 were collected at Paposo and Ovalle, Chile, respectively $7^{\circ}$ and $11^{\circ}$ latitude further south than guanaco138DA and 8, collected in Putre, northern Chile (Fig. 1b; Additional file 1: Figure S2). Guanaco 22 and 24 are therefore likely mislabelled as L. g. cacsilensis since they were collected within the "dry diagonal," an arid region and known biogeographic transition zone in the Andean chain [12, 13]. All llamas grouped with the two north Chilean L. g. cacsilensis, supporting the hypothesis that llamas were domesticated from within the L. g. cacsilensis gene-pool. In contrast, both currently described vicuña subspecies clustered separately, with $100 \%$ bootstrap support. $V . v$. mensalis were sampled in northern Chile and Peru, whereas $V$. v. vicugna were sampled in Chile and Argentina. Despite clustering within Vicugna, and with $100 \%$ bootstrap support, the alpacas did not cluster within either the $V . v$. mensalis or $V$. v. vicuna groups, instead forming a set of apparently divergent lineages with longer branch lengths, nested within the broader Vicugna cluster. However, after Local Ancestry Inference (LAI)-assisted removal of all alpaca genomic regions not having significant vicuña ancestry (Fig. 1a lower, see below), the relative position of alpaca changed to group with $V . v$. mensalis, consistent with a northern origin of domestication of the alpaca as previously reported $[3,7]$.

Admixture cross-validation analysis showed the optimal grouping to be $k=2(\mathrm{CV}=$ $0.593,0.620,0.657,0.744$ for $k=2,3,4,5$ respectively). At $k=2$, the results were broadly concordant with the neighbor-joining tree (Fig. 1c), with llama clustering with guanaco and alpaca with vicuña. However, alpaca showed evidence for strong 


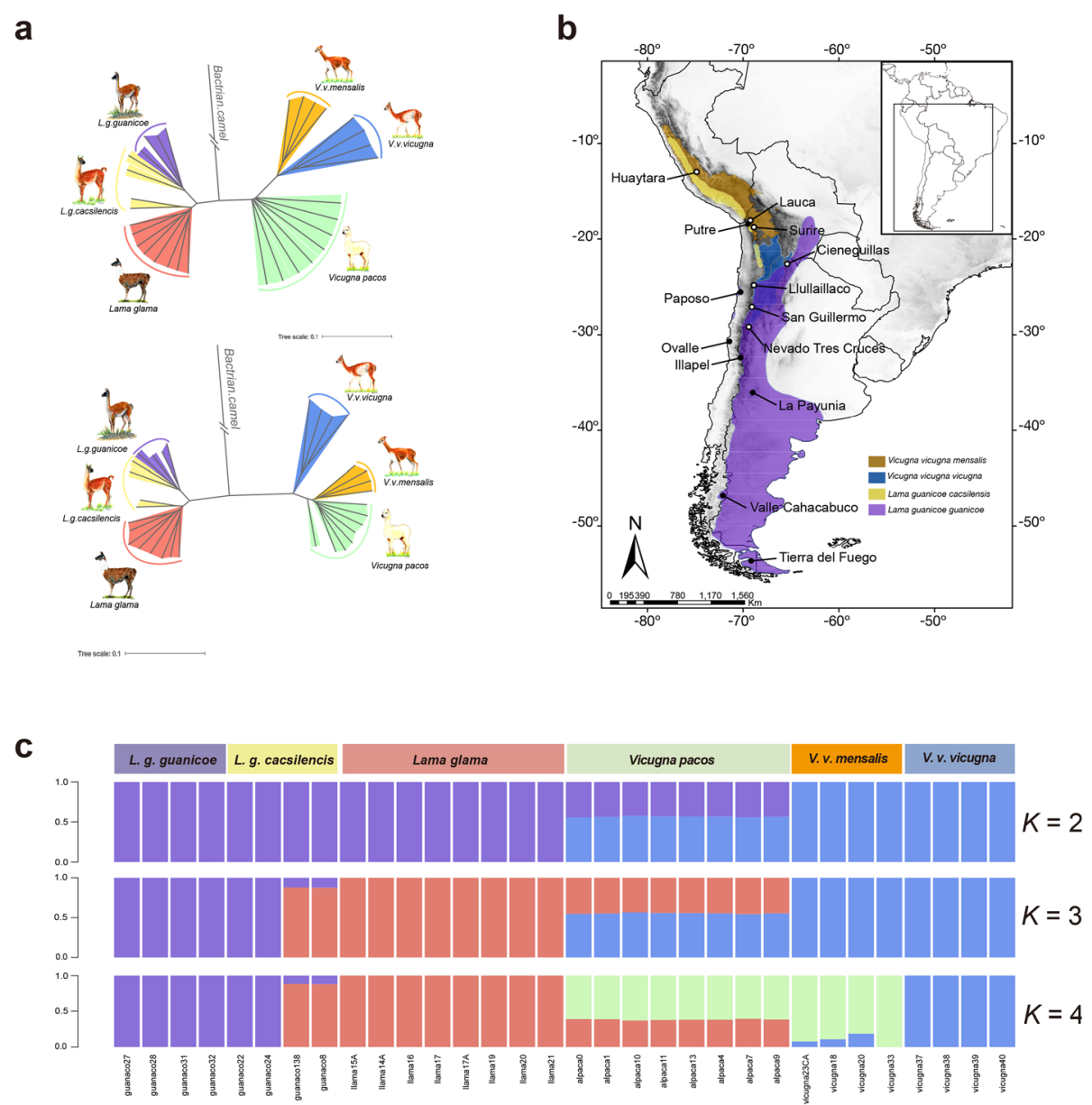

Fig. 1 Evolutionary relationship among South American camelids (SACS). a Neighbor-joining (NJ) trees by using all whole-genome data (top) and by using loci located in alpaca regions of highest vicuña ancestry (bottom). b Map with current distributions of SACs and sample location in this study. Open and solid dots indicate sampled localities of vicuña and guanacos respectively. c Model-based clustering of 32 SACs performed using ADMIXTURE with the number of ancestry kinships (k) set to 2, 3, or 4. Each vertical bar in proportions is corresponding to its proportion of genetic ancestry from each of these ancestral populations. The names of species and samples are shown in top and below of figure respectively

admixture with approximately $36 \%$ of genomic SNPs detected in the genomes of all individuals analyzed clustering with guanaco/llama. At $k=4$ (Fig. 1c), the signal of admixture in the alpaca was still strongly evident, with the remainder of the genome clustering with $V$. v. mensalis, in accordance with Fig. 1a lower, and evidence of unidirectional admixture from llama to alpaca and from $V$. v. vicuna into mensalis. In addition, also in support of the neighbor-joining results, L. g. cacsilensis samples guanaco138DA and 8 clustered with all llamas (albeit with some evidence of admixture). Treemix results (Additional file 1: Figure S3) confirmed both the close relationship between V.pacos and V.v. mensalis and the unidirectional gene flow between llama and alpaca.

ABBA-BABA analysis produced a significantly positive $D$ value for ((alpaca, vicuña), llama, bactrian) of $0.565 \pm 0.006$ (two-tailed $z$-test, $P<0.01$ ), indicating strong genomewide introgression between llama and alpaca, recapitulating the results of the ADMIXTURE and Treemix analysis. In contrast, we found a significantly negative $D$ value for ((guanaco, llama), vicuña, bactrian) of $-0.185 \pm 0.006$ (two-tailed $z$-test, $P<0.01$ ), 
suggesting the introgression from alpaca into llama was significantly lower ( $t$-test, $P<$ 0.001 ). The introgression proportion identified by using $f_{d}$ analysis was $\sim 38.9 \%$ from llama to alpaca and $\sim 4.5 \%$ in the opposite direction (Fig. 2a, b; Additional file 1: Table S10). Local Ancestry Inference estimates of ancestry proportions for llama and alpaca were highly concordant with $f_{\mathrm{d}}$ and ADMIXTURE results, with approximately $94.5 \%$ of llama ancestry and $36 \%$ of alpaca ancestry inferred to have originally originated from guanaco/llama and 5.5\% and 64\% derived from vicuña/alpaca, respectively (Fig. 2a, b). We identified 290 high probability llama ancestry introgressed windows (top 1\%, size = $100 \mathrm{~kb}$ ) using LAI or $f_{\mathrm{d}}$, comprising 225 and 236 genes, respectively (Fig. 3a; Additional file 1: Table S11). There were 87 windows (comprising 101 genes) detected in common by both methods (Additional file 1: Table S11), which were investigated for GO terms (Table 1). Using the same approach, we identified 247 high alpaca ancestry regions using LAI or $f_{\mathrm{d}}$, comprising 335 and 287 genes, respectively (Additional file 1: Table S11; Figure S4) with 113 regions (192 genes) detected in common (Additional file 1: Table S12), which were also investigated for GO terms. GO analysis (Table 1) identified regions including olfactory receptor genes, putative blood pressure markers, fleece quality and color genes (bidirectional introgression), and disease resistance/susceptibility (unidirectional introgression).

We examined introgression patterns along the X-chromosome in comparison to the autosomes. For segments where we could unambiguously identify contiguous Xchromosomal regions, we found relatively low $D$ and $f_{\mathrm{d}}$ values (see Fig. 3b) of $0.278 \pm$ 0.414 (s.d) and $-0.001 \pm 0.435$ for llama to alpaca and alpaca to llama, respectively, compared to $0.560 \pm 0.203$ and $0.172 \pm 0.234$ for the autosomes ( $t$-tests for both comparisons $P<0.001$; Fig. $3 \mathrm{~b}$ ) allowing us to infer significantly lower introgression into the $\mathrm{X}$ chromosome, implying an adaptive cost of introgression between llama and alpaca as has been seen in other domestic hybrids [27] or strong ancestral selective sweeps in the region, inhibiting our ability to detect introgression because of low divergence [28]. Inferred introgression was especially variable on the $\mathrm{X}$-chromosome, which, after filtering for putative Y-chromosome and pseudo-autosomal regions (PARs), featured some regions of high introgression (four regions comprising 79 genes) but also regions of almost zero introgression (four regions comprising 116 genes) (Fig. 3c). The eight regions contained genes with known deleterious or lethal X-linked effects in humans or mouse (25 genes in low introgression regions and 28 in high regions). For example, the deleterious mutations in the PTCHD1 (patched domain containing 1) gene have been described in male patients with X-linked autism spectrum disorder (ASD) and/or intellectual disability (ID) [29, 30], which further been found that the deficiency of PTCHD1 could also induce excitatory synaptic and cognitive dysfunctions in mouse [31]. Beyond it, eight genes feature dominant inheritance, five of unknown or variable inheritance and 12 recessive for low regions and eight, nine, and 11 such genes found in highly introgressed regions (Additional file 1: Table S13). Taken together, these results suggest that selection could be limiting heterogametic introgression of deleterious variants between Lama and Vicugna in domestic crosses.

Analysis of incomplete lineage sorting (ILS) indicated a higher ILS rate for llamaguanaco-alpaca (0.275) than for llama-guanaco-vicuña (0.157), which in the absence of admixture would be expected to give similar estimates if the alpaca is descended from the vicuña. Within each species trio, the two different ILS topologies would also be 


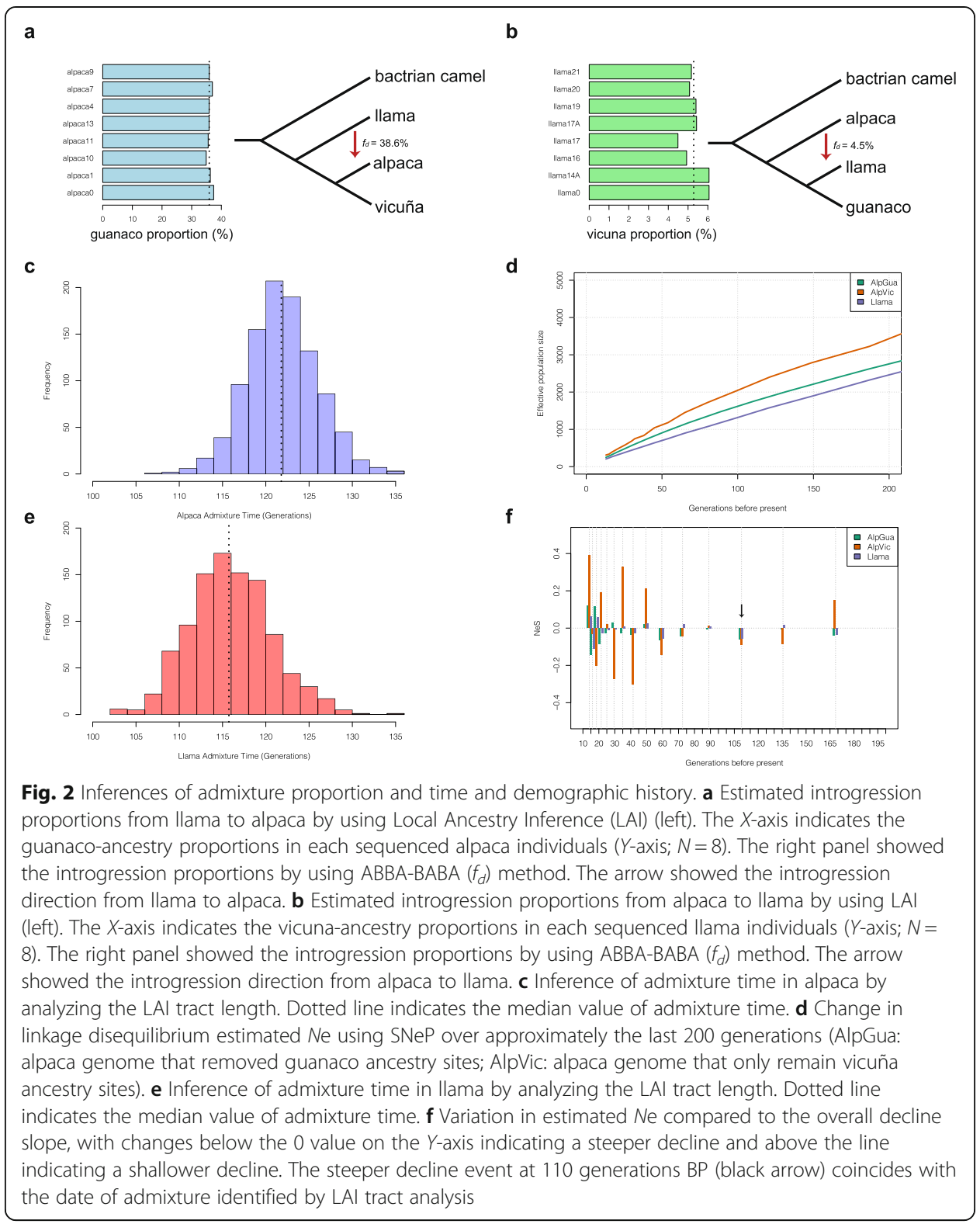

expected to appear in equal proportions in the absence of admixture. However, we found a larger proportion of ((llama, alpaca), guanaco) (0.1480326) compared to ((guanaco, alpaca), llama) ILS (0.124) and a larger proportion of ((llama, vicuña), guanaco) (0.092) compared to ((guanaco, vicuña), llama) ILS (0.062). This larger proportion of ILS involving llama further suggests admixture between alpaca and llama.

Using LAI admixture tract length analysis, we obtained very similar estimates of admixture time for both domesticated species, with 121.17 generations for alpacas (113.99-130.04 95\% CI; Fig. 2c) and 115.13 generations for llamas (107.4-126.02 95\% CI; Fig. 2e). The generation length of llamas and alpacas has not been reported, although some widely divergent estimates are available for guanaco varying between 3 and 11 years [32,33]. We assumed that domesticated species would have shorter generation lengths than their wild counterparts, a common feature of domestication and selective breeding and loss of seasonality [27, 34]. Given the range of guanaco estimates 


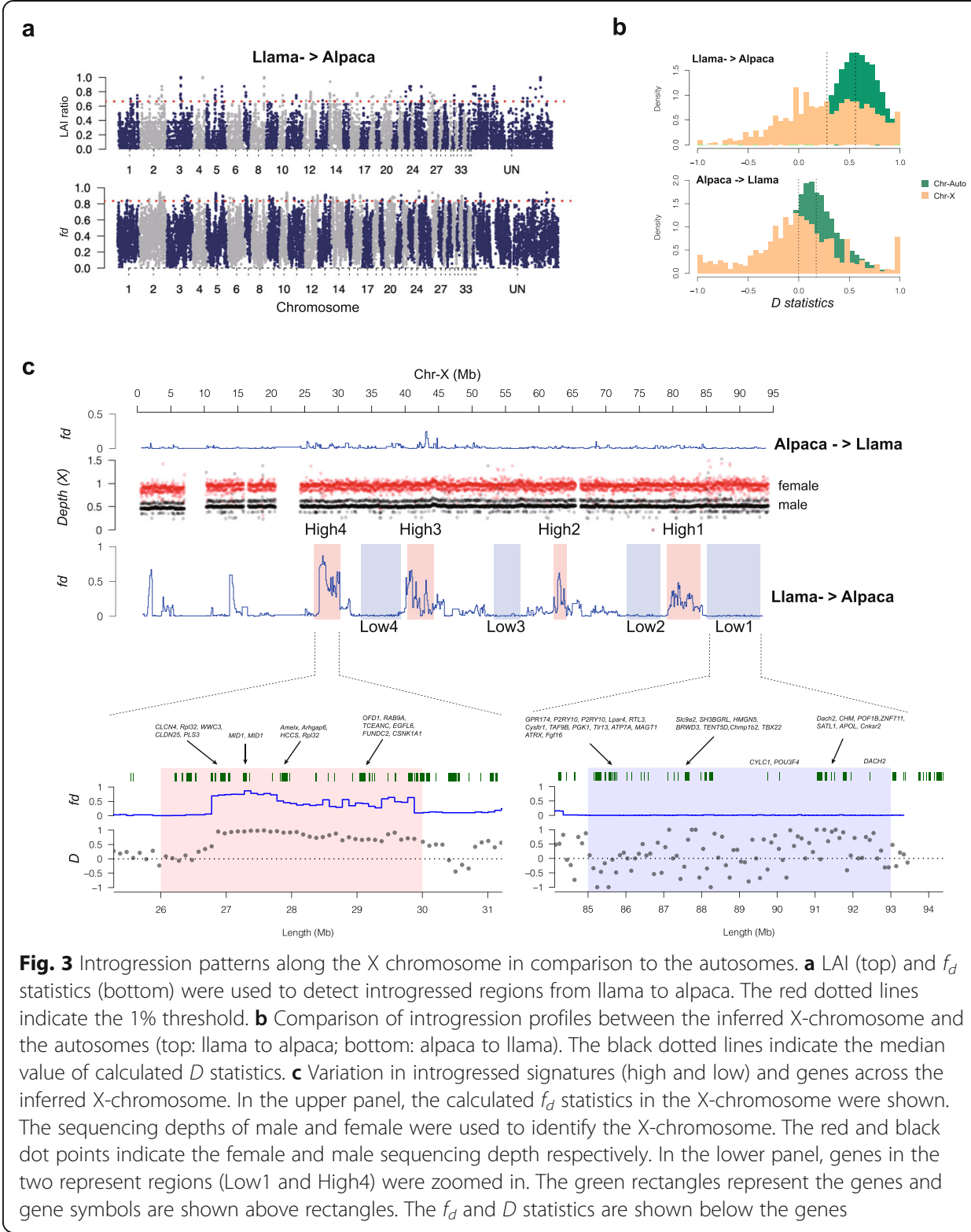

available, the implications of the LAI results for 3, 4, 5, and 6-year generation lengths (the mean estimate for guanaco being 7 years) are events occurring at 345, 461, 575, and 691 years ago, respectively, for llama and 364, 485, 606, and 727 years ago for alpaca. The most plausible explanation for a contemporaneous single-pulse admixture event occurring for both domestic species in this geographic region within this timeframe is the arrival of the Spanish Conquistadors (starting 1529). If this event explains the admixture tracts within the genomes of the domestic species, we can infer a generation length for both species at approximately 4 years.

\section{Demographic history}

PSMC (Pairwise Sequential Markovian Coalescent) showed signatures of a strong demographic expansion from one million years before present (BP) for vicuña and 
Table 1 Genes, location, inferred or potential function, and directionality of introgression between Ilama and alpaca, revealed by Local Ancestry Inference and ABBA-BABA analysis $\left(f_{\mathrm{d}}\right)$

\begin{tabular}{|c|c|c|c|c|}
\hline Gene & Chromosome & Function & Directionality & $\begin{array}{l}\text { LAl, } f_{\mathrm{d},} \\
\text { or both }\end{array}$ \\
\hline $\begin{array}{l}\text { Olfactory receptor (OR5) } \\
\text { family }\end{array}$ & ChruN & Olfaction/dietary choice [14] & $\begin{array}{l}\text { Llama- } \\
\text { Alpaca }\end{array}$ & Both \\
\hline $\begin{array}{l}\text { Anthrax toxin receptor } 2 \\
\text { (ANTXR2) }\end{array}$ & 2 & Anthrax resistance/susceptibility [15] & $\begin{array}{l}\text { Llama- } \\
\text { Alpaca }\end{array}$ & Both \\
\hline $\begin{array}{l}\text { Fibroblast growth factor } 5 \\
\text { (FGF5) }\end{array}$ & 2 & Longwool/shortwool fleece [16] & $\begin{array}{l}\text { Llama- } \\
\text { Alpaca }\end{array}$ & Both \\
\hline C4orf22 & 2 & $\begin{array}{l}\text { ANTXR2,FGF5, C4orf22 region linked to } \\
\text { high blood pressure susceptibility [17] }\end{array}$ & $\begin{array}{l}\text { Llama- } \\
\text { Alpaca }\end{array}$ & Both \\
\hline $\begin{array}{l}\text { Atrial natriuretic peptide- } \\
\text { converting enzyme (CORIN) }\end{array}$ & 2 & $\begin{array}{l}\text { Negatively regulates Agouti, yellow coat } \\
\text { in Hanwoo cattle [18] }\end{array}$ & $\begin{array}{l}\text { Alpaca- } \\
\text { Llama }\end{array}$ & Both \\
\hline Endothelin 3 (EDN3) & 19 & $\begin{array}{l}\text { Blue-eyed, white coat phenotype in } \\
\text { Waardenburg syndrome of human [19] }\end{array}$ & $\begin{array}{l}\text { Alpaca- } \\
\text { Llama }\end{array}$ & Both \\
\hline $\begin{array}{l}\text { Olfactory receptor (OR2) } \\
\text { family }\end{array}$ & Chrun & Olfaction/dietary choice [14] & $\begin{array}{l}\text { Alpaca- } \\
\text { Llama }\end{array}$ & $f_{d}$ \\
\hline $\begin{array}{l}\text { Fibroblast growth factor } 21 \\
\text { (FGF21) }\end{array}$ & 11 & Melanogenesis in alpaca [20] & $\begin{array}{l}\text { Alpaca- } \\
\text { Llama }\end{array}$ & Both \\
\hline $\begin{array}{l}\text { Agouti signaling protein } \\
\text { (ASIP) }\end{array}$ & 19 & Pigmentation $[21,22]$ & $\begin{array}{l}\text { Alpaca- } \\
\text { Llama }\end{array}$ & $f_{\mathrm{d}}$ \\
\hline Transgelin (TAGLN) & 33 & Fiber development in Merino sheep [23] & $\begin{array}{l}\text { Alpaca- } \\
\text { Llama }\end{array}$ & $f_{\mathrm{d}}$ \\
\hline GNAS complex & 19 & $\begin{array}{l}\text { Performance traits in cattle (imprinted) } \\
\text { [24] }\end{array}$ & $\begin{array}{l}\text { Alpaca- } \\
\text { Llama }\end{array}$ & Both \\
\hline Cathepsin Z (CSTZ) & 19 & Immunoprotection $[25,26]$ & $\begin{array}{l}\text { Alpaca- } \\
\text { Llama }\end{array}$ & Both \\
\hline
\end{tabular}

alpaca, while guanaco and llama showed an expansion only after c 400,000 years, having undergone a decline immediately prior to this expansion (Additional file 1: Figure S5). All species showed evidence of a subsequent strong decline from 100,000-200,000 years BP to 25,000 years BP, overlapping with the last two major glacial periods. The alpaca genome showed evidence of a stronger and longer expansion than the vicuña; however, an even more extreme expansion for alpaca (to effective size $N_{\mathrm{e}}<50,000$ ) was also detected in a previous study [8], but this study did not remove admixture tracts prior to analysis, potentially inflating the $N_{\mathrm{e}}$ estimates of the simulation [28]. To detect more recent $N_{\mathrm{e}}$ changes, we used MSMC (Multiple Sequence Markovian Coalescent), taking advantage of a sample of 6 (alpaca and llama) or 8 (vicugna and guanaco) resequenced genomes in this study and found concordant evidence for declines in all four species from 100,000 to 10,000 years BP. Higher $N_{\mathrm{e}}$ estimates could be inferred for $V$. $v$. vicugna compared to $V$. v. mensalis, consistent with their larger geographic range, but no such pattern was found for L. g. guanicoe compared to L. g. cacsilensis, with the variance among samples being larger than for Vicugna (Additional file 1: Figure S6). SNeP (SNP linkage disequilibrium-based $N_{\mathrm{e}}$ estimation), the resolution of which enables $N_{\mathrm{e}}$ trends and their variance to be inferred over the last 200 generations [35, 36], showed a consistent signature of further population decline (Fig. 2d), with the largest decline detected in alpaca, both when only the most highly significant vicuña ancestry portions of the alpaca genome were included (43\% of the genome retained) and when only the most highly significant guanaco ancestry portions of the alpaca genome were removed (64\% retained). Estimated $N_{\mathrm{e}}$ values ranged from 2500 (llama) to 3500 (vicuña-ancestry 
alpaca), closely resembling those values from 1000 years BP in both species' MSMC simulations. NeS analysis [36] identified considerable variation in the $N_{\mathrm{e}}$ decline slope, especially in the recent past (50 generations), but interestingly identified an increase in the rate of decline for both domestic species at approximately 110 generations ago, which coincides closely with the LAI tract-based estimate of time of admixture (Fig. 2f).

\section{Selection signatures for domestication and introgressed segments}

Combining $F_{\mathrm{ST}}$ outlier and extended haplotype homozygosity (XP-EHH) analysis, we analyzed signatures of domestication by directly comparing wild ancestor and domestic descendant pairs (vicuña versus alpaca and guanaco versus llama). For this analysis, we did not use the genome data for which admixture tracts had been removed to enable us to compare introgression "landmark" signatures with domestication signatures in the same analysis. For vicuña versus alpaca, mean $F_{\mathrm{ST}}$ across the genome was $0.086 \pm 0.153$. For domestication signals in alpaca, we identified 92 and 128 regions, comprising 647 and 817 genes, using $F_{\mathrm{ST}}$ and XP-EHH, respectively (Additional file 1: Table S14-15; Figure S7). However, only four regions comprising 35 genes showed overlapping peaks between the two methods (Additional file 1: Table S16). Although overlap between the two methods was limited, aside from the expected introgressed segments inferred to have originated from llama, putative domestication regions were linked to fecundity/development of secondary sexual characters, coat color, hypoxia, meat quality, and olfaction [37] (Table 2). For guanaco versus llama, the mean $F_{\mathrm{ST}}$ across the genome was $0.064 \pm 0.128$. For domestication signals, we identified 141 and 126 regions, comprising 999 and 800 genes, using $F_{\mathrm{ST}}$ and XP-EHH, respectively (Additional file 1: Table S17-18; Figure S8). Fifteen regions comprising 79 genes showed overlapping peaks between the two methods (Additional file 1: Table S19). Although overlap between the two methods was again limited, aside from the expected introgressed segments inferred to have originated from alpaca, putative domestication regions were linked to blood pressure control, spatial memory and social behavior, skin and coat color, domestic performance traits, and dietary restriction and appetite [34] (Table 2).

\section{Evidence for adaptive introgression}

We then attempted to combine an understanding of both introgression and selection identified via the co-detected segments in our domestication analysis by focusing on four introgressed regions (ANTRX2/PRDM8/FGF5/C4orf22 and OR5 family for llama to alpaca, $E D N 3$ and OR2 family for alpaca to llama). We examined phased haplotypes along the inferred introgressed regions to confirm both their ancestry from vicuña or guanaco and the variance in $F_{\mathrm{ST}}$ between alpaca and llama to establish which segments showed the strongest signatures of selection (Fig. 4a-d).

For the ANTRX2/PRDM8/FGF5/C4orf22, the guanaco origin of the domestic haplotype is clearly evident, strongly supporting the inference that it was introgressed from the llama into the alpaca (Fig. $4 \mathrm{~b}$ ). Inspection of the $F_{\mathrm{ST}}$ distribution along the region shows a highly heterogeneous pattern, with almost no genetic differentiation between llama and alpaca coincident with 3' end of the C4orf22 gene and downstream (Fig. 4b). Further, SNP variation is, represented by genetic diversity $\left(\theta_{\pi}\right)$, as expected, highest in 
Table 2 Genes, location, inferred or potential function, and origin of domestication signatures in alpaca and llama, revealed by $F_{\mathrm{ST}}$ outlier and extended haplotype homozygosity (XP-EHH) analysis

\begin{tabular}{|c|c|c|c|c|c|}
\hline Gene & Chromosome & Function & Origin & $\begin{array}{l}\text { Domestic } \\
\text { species }\end{array}$ & $\begin{array}{l}F_{\mathrm{ST}}, \mathrm{XP}- \\
\mathrm{EHH} \text {, or } \\
\text { both }\end{array}$ \\
\hline $\begin{array}{l}\text { Anthrax toxin } \\
\text { receptor } 2\end{array}$ & 2 & $\begin{array}{l}\text { Anthrax resistance/susceptibility } \\
{[15]}\end{array}$ & $\begin{array}{l}\text { Introgressed } \\
\text { from llama }\end{array}$ & Alpaca & Both \\
\hline $\begin{array}{l}\text { Fibroblast growth } \\
\text { factor } 5\end{array}$ & 2 & Longwool/shortwool fleece [16] & $\begin{array}{l}\text { Introgressed } \\
\text { from llama }\end{array}$ & Alpaca & Both \\
\hline C4orf22 & 2 & $\begin{array}{l}\text { ANTXR2, FGF5, C4orf22 region } \\
\text { linked to high blood pressure } \\
\text { susceptibility [17] }\end{array}$ & Llama-Alpaca & Alpaca & Both \\
\hline $\begin{array}{l}\text { Luteinizing hormone } \\
\text { receptor }\end{array}$ & Chrun & $\begin{array}{l}\text { Fecundity and development of } \\
\text { male secondary sexual characters } \\
\text { [38] }\end{array}$ & Domestication & Alpaca & Both \\
\hline $\begin{array}{l}\text { Agouti signaling } \\
\text { protein }\end{array}$ & 19 & Pigmentation [21, 22] & Domestication & Alpaca & Both \\
\hline $\begin{array}{l}\text { Hypoxia upregulated } \\
1\end{array}$ & 33 & $\begin{array}{l}\text { Cytoprotection under hypoxic } \\
\text { conditions [39] }\end{array}$ & Domestication & Alpaca & $\mathrm{XP}-\mathrm{EHH}$ \\
\hline Myomesin 1 & 24 & $\begin{array}{l}\text { Meat quality in cattle, sheep, and } \\
\text { pigs [40-42] }\end{array}$ & Domestication & Alpaca & $F_{\mathrm{ST}}$ \\
\hline $\begin{array}{l}\text { Melanocortin } 5 \\
\text { receptor }\end{array}$ & 23 & Meat quality [43] & Domestication & Alpaca & Both \\
\hline $\begin{array}{l}\text { Melanocortin } 2 \\
\text { (adrenocorticotropic) } \\
\text { receptor }\end{array}$ & 24 & Circadial rhythm [44] & Domestication & Alpaca & $F_{\mathrm{ST}}$ \\
\hline $\begin{array}{l}\text { Melanocortin } 1 \\
\text { receptor }\end{array}$ & 24 & Light fiber color [45] & Domestication & Alpaca & $F_{\mathrm{ST}}$ \\
\hline $\begin{array}{l}\text { Olfactory receptor } \\
\text { (OR5) family }\end{array}$ & Chrun & Olfaction/dietary choice [14] & $\begin{array}{l}\text { Introgression } \\
\text { from llama }\end{array}$ & Alpaca & $F_{\mathrm{ST}}$ \\
\hline $\begin{array}{l}\text { Olfactory receptor } \\
\text { (OR2) family }\end{array}$ & Chrun & Olfaction/dietary choice [14] & Domestication & Alpaca & $F_{\mathrm{ST}}$ \\
\hline $\begin{array}{l}\text { G protein-coupled es- } \\
\text { trogen receptor } 1\end{array}$ & Chrun & $\begin{array}{l}\text { Spatial memory and social } \\
\text { behavior [46] }\end{array}$ & Domestication & Llama & Both \\
\hline $\begin{array}{l}\text { Premelanosome } \\
\text { protein }\end{array}$ & Chrun & $\begin{array}{l}\text { Skin and coat color in wild and } \\
\text { domestic mammals [47] }\end{array}$ & Domestication & Llama & $F_{\text {ST }}$ \\
\hline $\begin{array}{l}\text { Anthrax toxin } \\
\text { receptor }\end{array}$ & 2 & $\begin{array}{l}\text { Anthrax resistance/susceptibility } \\
{[15]}\end{array}$ & Domestication & Llama & Both \\
\hline $\begin{array}{l}\text { Fibroblast growth } \\
\text { factor } 5\end{array}$ & 2 & Longwool/shortwool fleece [16] & Domestication & Llama & Both \\
\hline Endothelin 3 & 19 & $\begin{array}{l}\text { Blue-eyed, white coat phenotype } \\
\text { in Waardenburg syndrome of } \\
\text { human [19] }\end{array}$ & $\begin{array}{l}\text { Introgression } \\
\text { from alpaca }\end{array}$ & Llama & $F_{\mathrm{ST}}$ \\
\hline $\begin{array}{l}\text { Agouti signaling } \\
\text { protein }\end{array}$ & 19 & Pigmentation $[21,22]$ & $\begin{array}{l}\text { Introgression } \\
\text { from alpaca }\end{array}$ & Llama & $\mathrm{XP}-\mathrm{EHH}$ \\
\hline GNAS complex & 19 & $\begin{array}{l}\text { Performance traits in cattle } \\
\text { (imprinted) [24] }\end{array}$ & $\begin{array}{l}\text { Introgression } \\
\text { from alpaca }\end{array}$ & Llama & $F_{\mathrm{ST}}$ \\
\hline $\begin{array}{l}\text { Agouti-related } \\
\text { neuropeptide }\end{array}$ & 9 & $\begin{array}{l}\text { Dietary restriction and appetite in } \\
\text { cattle [37] }\end{array}$ & Domestication & Llama & $F_{\mathrm{ST}}$ \\
\hline
\end{tabular}

guanaco, followed by llama and with alpaca nearly devoid of SNPs in this region $\left(\theta_{\pi^{-}}\right.$ guanaco $=0.17 ; \theta_{\pi}$-llama $=0.13 ; \theta_{\pi}$-alpaca $=0.02$ ), suggesting serial bottlenecks and $/$ or selection occurring first at the domestication stage (guanaco-llama, where $F_{\mathrm{ST}}$ and XPEHH scores were 0.106 and 0.269 , respectively) and second at the introgression stage (llama-alpaca, where LAI and $f_{d}$ scores were 0.912 and 0.867 , respectively). In contrast, for the EDN3 gene, while the alpaca origin of the domestic haplotype is clear (Fig. 4c), 
supporting its vicuña origin, there is no evidence for a loss of diversity between vicuña and alpaca (EDN3 does not feature in either the $F_{\mathrm{ST}}$ or XP-EHH candidate list; Fig. 4a) or at the stage of admixture (alpaca-llama, where LAI and $f_{d}$ scores were 0.400 and 0.664, respectively), suggestive of recent artificial selection for this phenotype in the domestic forms (white fleece, blue eyes [48]; Fig. 4e). In both alpacas and llamas, white individuals with blue eyes are often deaf, the phenotype of which is known to be regulated by KIT [35]. However, in the phenotypically similar Waardenburg syndrome in humans, these phenotypes are associated with EDN3, SOX10, and EDNRB [19]. While the linkage of these phenotypes to $E D N 3$ in SAC was previously unsuspected, studies have suggested EDN3 is linked to white pelage in wild cats [49] and the cooccurrence of deafness (congenital deafness) and white pigmentation is a common observation in domestic animals [50].

We also found that the number of SAC functional olfactory receptor $(O R)$ genes was substantially lower than for other domestic livestock (approximately $50 \%$ of cattle and horse, at 566-602; Additional file 1: Table S20). For OR5, while the wild origin of the haplotype (especially $5^{\prime}$ end) in the guanaco was clear for both domestic forms, a mosaic pattern of similarity is evident across the family, with no consistent changes in $F_{\mathrm{ST}}$ being evident (Additional file 1: Figure S9 left). For OR2, a vicuña origin is clear, but again, no consistent patterns are discernible across the region (Additional file 1: Figure S9 right).

Given the extensive admixture detected between llama and alpaca, a wider investigation of introgressed segments was carried out (HoxD cluster, EVX2, OLA1, and a 1.5 $\mathrm{Mb}$ region on Chr10 from llama to alpaca, including C2CD3, RAB6C, ATG16L2, and RNF121; CORIN, FGF21, GNAS, and TAGLN from alpaca to llama; see Additional file 1: Figure S10-12). For HoxD (HoxD3, HoxD4, HoxD10, HoxD12, and HoxD13), EVX2 and $O L A 1$, we found clear evidence of introgression from llama to alpaca (Additional file 1: Figure S10). Mutations in $\operatorname{HoxD}$, EVX2, and $O L A 1$ genes are known to result in abnormal skeleton development [51, 52] and introgression of $\operatorname{HoxD}, E V X 2$, and $O L A 1$ from llama to alpaca could therefore influence the development of the alpaca's larger body size compared with vicuña, as evidenced, for example, by obvious shoulder height differences [53] (Additional file 1: Figure S10-12).

\section{Functional analysis}

We found that the coding sequences (CDS) for FGF5 in male and female Suri and Huacaya alpaca skins were identical except for two $T$ - G substitutions at sites 50 and 346 (Additional file 1: Figure S13a). To establish whether FGF5 was expressed differentially in the skin of Suri (longwool) and Huacaya (shortwool) alpaca, quantitative real-time PCR (qPCR) analysis was carried out and showed that FGF5 mRNA showed significantly elevated expression in Suri alpaca, both for males and females (two-way ANOVA $P<0.001$ ) (Additional file 1: Figure S13b), a result replicated in concomitant Western Blot assays (two-way ANOVA $P<0.001$ ) (Additional file 1: Figure S13c, d). Expression profiles for the 20 single-copy genes identified during the introgression and selection signature analysis were compared within alpaca and among the three de novo sequenced specimens. We found very high expression levels for transgelin (7x higher compared to all other genes) in alpaca and llama skin (expressed at a similar level to 


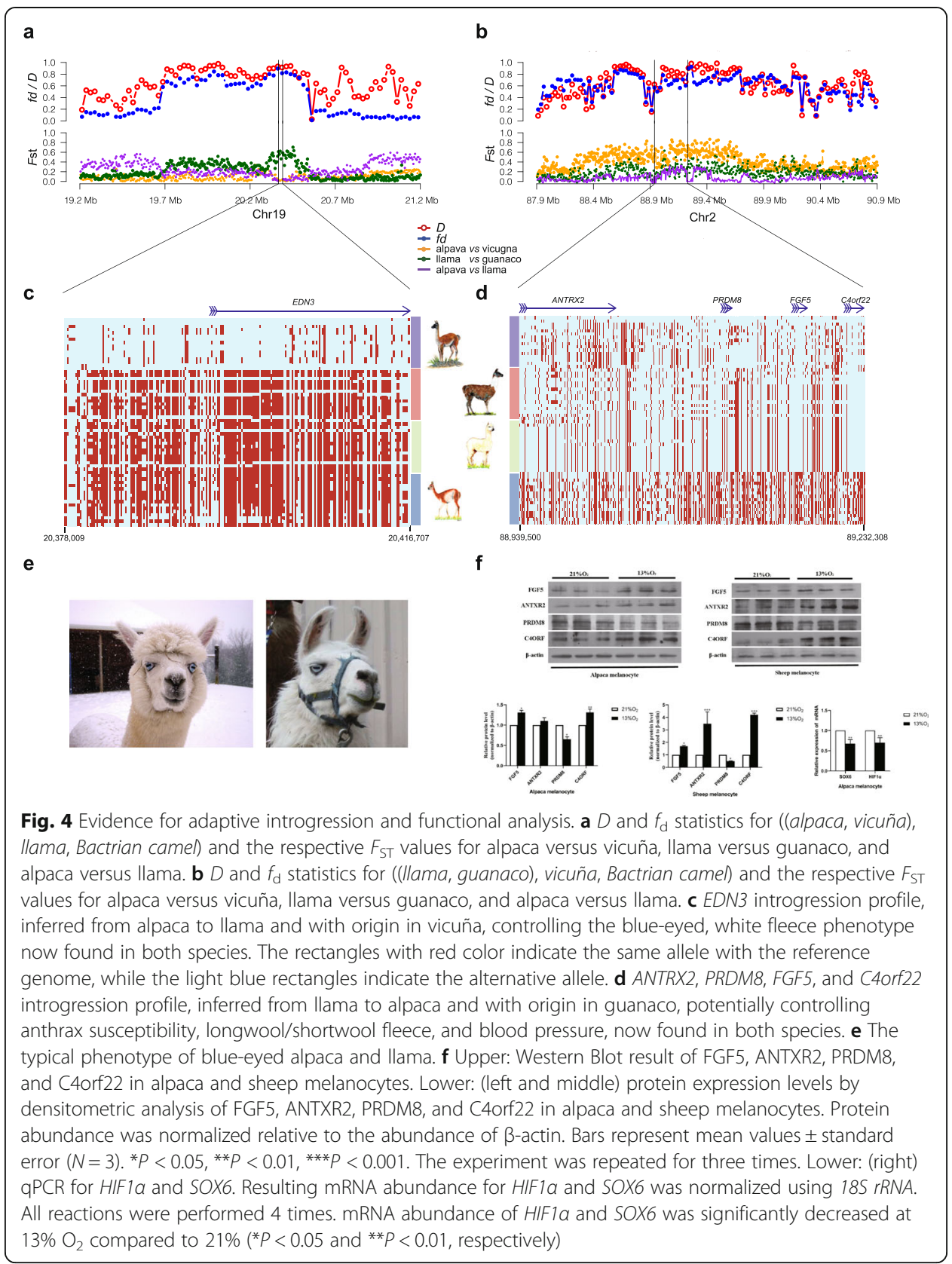

alpaca, although in blood, while being effectively absent from the wild species). This gene was identified as being introgressed from alpaca to llama has been linked to skin and wool follicle development in merino fine-wool sheep [23]. To investigate whether FGF5, ANTXR2, PRDM8, C4orf22 haplotype could be related to hypoxic response, as some evidence has already been found in other mammals [54, 55], their expression was investigated in alpaca and sheep melanocytes incubated under normoxic $\left(21 \% \mathrm{O}_{2}\right)$ and hypoxic $\left(13 \% \mathrm{O}_{2}\right)$ conditions. While FGF5, ANTXR2, and C4orf22 were more highly expressed in hypoxic conditions, surprisingly $P R D M 8$ at $13 \% \mathrm{O}_{2}$ was significantly less expressed than at $21 \% \mathrm{O}_{2}$ in both cell lines. Similarly, mRNA expression of Sry-related HMG-box-containing transcription factor 6 (SOX6) and its downstream regulated 
hypoxia-related gene $H I F 1 \alpha$ [56] were downregulated at $13 \%$ compared to those at $21 \%$ $\mathrm{O}_{2}$ (Fig. 4f).

\section{Discussion}

These first genome-scale results for wild and domestic South American camelids convincingly support the hypothesis that the alpaca was domesticated from the vicuña and that the llama was domesticated from the guanaco. Figure $1 \mathrm{~b}$ shows that despite the wide spread of alpaca lineages in the Vicugna clade, they group together with 100\% bootstrap support, despite the $>30 \%$ introgression from llama that was detected. When we recalculated the phylogeny with the introgression segments removed from the alpaca genome (Additional file 1: Figure S14), all individuals grouped together and with $V$. v. mensalis with $100 \%$ bootstrap support, pointing towards this subspecies as the origin of the alpaca. Interestingly, however, all alpaca grouped within a separate clade from mensalis, suggesting that the population of $V . v$. mensalis that was originally domesticated is not covered by our mensalis samples. It remains possible, however, that residual introgression segments which we were unable to reliably detect using LAI have influenced the shape of the phylogeny and could underlie the separation of alpaca from mensalis (Fig. 1a). Figure 1a also reveals that Lama guanicoe cacsilensis is most similar to L. glama, which is recapitulated in Fig. 1c, where the two northern cacsilensis samples group with llama $(k=4)$. However, while we consider the phylogenetic origins of these domestic species to be resolved, we did not attempt to address how many times each species was domesticated nor the likely precise origins of domestication, which will require greater sampling of extant populations, especially in Peru, combined with an ancient DNA approach.

The level of admixture detected in the genomes of all alpacas sequenced is among the highest detected in a domesticated animal to date: all estimates exceeded $30 \%$. Figure 1a shows the admixture to be pervasive and remarkably consistent across individuals, implying that admixture happened in a consistent manner. Although a much smaller proportion of the llama genome is admixed (c 5\%), hybridization has clearly been bidirectional. All methods gave similar results but the LAI analysis of admixture tract lengths (Fig. 2c, e) allowed us to conclude that a single major event occurred contemporaneously for both species and is relatively recent, occurring just over 100 generations ago, consistent with the low variance in ancestry components detected [57]. While a precise estimate of the llama and alpaca generation length is not available, estimates using data from wild SACs would place the event at 400-600 years ago. The one major event that is known to have occurred during this period was the Spanish conquest. Within a century of the conquest of Cuzco in 1532, administrative and taxation documents record the "precipitous decline and virtual disappearance of previously extensive herds throughout the Andes", and while early Spanish writers noted the importance of SACs in the Andean pastoral system, they failed to distinguish between the llama and alpaca in their writings [3]. At a time of mass human and SAC mortality and replacement, under a subjugate system that failed to acknowledge the distinctiveness of the two domestic species, and with indigenous records and knowhow in breeding being passed down by oral tradition only, it seems likely that in the years after the conquest, established breeding practices broke down, including the maintenance of species boundaries and fleece types. 
The demographic history of SACs since the divergence of Lama and Vicugna c $2.5 \mathrm{M}$ years before present shows evidence for a strong expansion, albeit earlier for the vicuña, with the expansion of the genus Lama coinciding with the Mindel-Riss/Holstein interglacial period followed by a decline over the last 200,000 years, which has continued largely to the present day (Additional file 1: Figure S5 and S6). The difference between the two genera may reflect their altitudinal partitioning and reliance of the guanaco on multiple Andean habitats, which would have opened up during this interglacial period, potentially allowing the population to expand. These results are interesting in the context of recent studies on the genetics of the wild species $[57,58]$ that have shown both a signature of demographic expansion in vicuña and guanaco and a recent demographic decline in vicuña, and our results support the latter conclusion [52]. However, the multi-locus microsatellite data used in these studies did detect a strong bottleneck 2000-3000 years ago, which coincides the period of strongest decline in our MSMC plots (Additional file 1: Figure S6). We were most interested in understanding finescale changes in effective population size in the last 1000 years for the domestic species and $\mathrm{SNeP}$ and $\mathrm{NeS}$ analysis both confirmed a continuing decline from 200 generations ago to the present day (Fig. 2d), to a current effective size of less than 1000. Interestingly, a coincident acceleration in this decline was detected approximately 110 generations ago using $\mathrm{NeS}$ (Fig. 2f), very close to the date estimated for the admixture pulse and in line with the recorded decline following the Spanish conquest [3]. So, taken together, the admixture tract and $\mathrm{SNeP}$ analysis point to a single event in both species occurring 100-110 generations ago. The most plausible explanation for this decline in population size and the onset of substantial bidirectional introgression in these domestic species is the chaos and loss of traditional management practices in Andean communities immediately after the conquest.

The phenotypic consequences of the admixture pulse have never been studied, despite evidence of its importance. Annotation of the introgressed regions (Table 1) revealed several large haplotypes containing a number of genes that could be both separately or coincidentally introgressed. These include a haplotype including the $A n$ thrax toxin receptor 2 gene (ANTXR2) and fibroblast growth factor 5 (FGF5 - introgressed from llama to alpaca). This haplotype has been implicated in fiber characteristics in alpaca and blood pressure control in humans (Table 1) and Bacillus anthracis is also known to be prevalent in the soil at elevations below $4000 \mathrm{~m} \mathrm{ASL}$ in the Andes, but to be absent above this altitude [59]. Other genes detected included olfactory receptor families 2 and 5 (OR2 and OR5), genes linked to dietary choice [14] and coat color. The extent to which this introgression was deliberate or occurred accidentally is not known and merits further study, especially using historical records.

Signatures of domestication, identified by comparing the genomes of the domestic species with their wild ancestors (Table 2), as expected highlighted genes involved in coat color variation, sexual and reproductive traits, and dietary choice as well as a number of genes implicated in production traits (both meat and milk) in other livestock species. Interestingly, the alpaca whose wild ancestor, vicuña, lives at high altitudes, showed selection signatures for the hypoxia upregulated gene [39], while llama (whose wild ancestor, the guanaco, lives in small social groups and undertakes seasonal altitudinal migration) showed a signature for a G-coupled estrogen receptor previously linked spatial memory and social behavior [46]. As expected, the inferred introgressed 
segments described above were also identified as domestication signatures, implying that some are operating in parallel in the two domestic forms.

To further investigate the adaptive or selective value of the most significant introgressed segments in this study, we evaluated their sequence diversity in more detail. First, while admixture has been bidirectional, the generally lower levels of introgression in the X-chromosome suggest a potential barrier to hybridization which might have further impacted on effective population size trajectory after the conquest. This phenomenon has been observed in comparisons between taurine and indicine cattles [60], but the ancestors of those two bovine forms are generally thought to have been subspecies, not species from separate genera, for which the cost of introgression is expected to be considerably higher.

Analysis of the sequences that is syntenic to the human HSA4q21 locus comprising ANTRX2/PRDM8/FGF5/C4orf22 (llama-alpaca), EDN3 (alpaca-llama), and the OR5 and $O R 2$ gene families revealed contrasting patterns of genetic erosion, ranging from the expected serial loss of SNP diversity from wild ancestor to domesticated descendant and then into the introgressed species (see $A N T R X 2$; Fig. 4d), to very little difference detected in SNP diversity across all three species (EDN3), to a complex mosaic of SNP diversity across the $O R$ families though we cannot rule out the possibility that some $O R$ SNPs may be artifacts due to misaligned sequences. Our results thus suggest multiple evolutionary processes at play and the need to examine their evolution on a case-bycase basis. The evolutionary distinctiveness of the wild ancestors of these two domestic forms means that detecting introgression events is feasible; however, understanding their functional significance is less straightforward than, for example, can be carried out within and among livestock breeds within the same species which have an extensive history of QTL investigation, e.g., in cattle [61]. Intriguingly, the HSA4q21 locus has been linked using GWAS, to blood pressure homeostasis in Asians and Europeans. A mouse model has been used to identify the causative gene in humans, which was found to be ANTXR2, whereas blood pressure did not differ between FGF5 knockout and wild-type mice [17, 62]. Systematic studies have identified a positive link between altitude (and thus hypoxia) with the frequency of hypertension in humans [63] and PRDM8 belongs to a family of histone methyltransferases that are known as negative regulators of transcription. Thus, in contrast to the other upregulated genes in the "HSA4q21" region, the downregulation of PRDM8 at high altitude may confer cytoprotection by upregulating the expression of other genes in a manner analogous to the hypoxia-inducible factor gene (e.g., HIF2 $\alpha$ ) [64]. Our qPCR results showed that mRNA expression levels for the genes SOX6 and HIF1 $\alpha$ are similarly downregulated. SOX6 is one of the targets of PRDM8 in the retina and its expression is controlled by PRDM8 [65]. The response of HIF1 $\alpha$ may therefore be indirect via the expression of the transcription factors SOX6 (and/or SOX5 and SOX9), depending on levels of atmospheric oxygen availability [56]. Under hypoxia, mitochondrial $\mathrm{O}_{2}$ consumption is inhibited or intracellular Fe and prolyl hydroxylase domain (PHD) activity increases, which decreases the expression of HIF1 $\alpha$ [56]. Therefore, FGF5, ANTXR2, and C4orf22 may play a key role in regulating hypoxia stress, while $P R D M 8$ is a novel gene associated with hypoxic adaptation, the expression of which may be mirrored by decreased expression of SOX6 and HIF1 $\alpha$. FGF5 was originally reported as a key human oncogene [66], associated with a number of cancers [67], angiogenesis in human aortic endothelial cells 
[68], and trichomegaly in humans [69]. C4orf22 is expressed in human neural progenitor cells expressing MEF2CA [70]. Functional analysis of the FGF5 gene in Suri and Huacaya alpacas has previously shown its potential importance in regulating fleece length [71], and preliminary analysis of expression profiles in domestic SACs has shown the potential importance of a novel QTL for fine fiber production in merino sheep [23]. Further studies should focus on investigating these genes and the QTL they underpin.

\section{Conclusions}

That domesticated South American camelids have undergone genetic erosion is clear from the decline in both species' effective sizes over the recent 1000 years, and it is plausible that the Spanish conquest and its aftermath have contributed to this substantially. However, it is also the case that some of the introgressed segments we have identified have potentially allowed new avenues for selection, for example, for coat color, fiber characteristics, and adaptation to high altitude and harsh environment. Therefore, introgression, which has negative connotations in species management or conservation, but which genome data are showing to be far more pervasive than previously appreciated, should be afforded a more nuanced interpretation in domestic species and does not necessarily contribute to genetic erosion.

\section{Methods}

\section{Sampling and DNA sequencing}

Blood and tissue samples were obtained from eight wild guanacos, vicunas, and domestic llamas and from seven alpacas. Sampling of guanacos and vicuñas represents the entire range of geographic distribution of each species (Additional file 1: Table S8). One adult male guanaco from Putre (Chile) and adult female vicuña from Lauca National Park (Chile) were selected for de novo assembly, representing the northern subspecies of wild South American camelids, Lama guanicoe cacsilensis and Vicugna vicugna mensalis, respectively. Llama tissues were obtained from Putre and alpaca from Temuco (Chile; Additional file 1: Table S8). Genomic DNA was extracted using the Puregene Tissue Core Kit A (Qiagen). See Additional Material S3 for further information and permit details.

\section{De novo assembly, alignment, and annotation}

De novo assembly for L. guanicoe, V. vicugna, and L. glama used Illumina HiSeq 2000 and HiSeq 2500 platforms (see Additional file 1: Supplementary text for full description). Paired-end libraries with insert sizes of approximately 170, 500, and $800 \mathrm{bp}$, and mate pair libraries with insert sizes of 2, 5, 10, 20, and $40 \mathrm{~kb}$ were constructed. We generated 335, 285, and $262 \mathrm{~Gb}$ of high-quality data for each species, respectively (Additional file 1: Table S1). SOAPdenovo (v2.04) [9] was used to assemble the genomes (Additional file 1: Table S2). Annotation used a standard combination of ab initio gene prediction, homolog searching, and EST/unigene-based prediction. To aid gene prediction and to enable transcript data to be analyzed at the species level, we generated RNA-seq data for blood samples from the vicuña, llama, and guanaco used for de novo sequencing and from skin samples for a set of 17 alpaca samples from China 
(Additional file 1: Table S8). Expression patterns were compared within and among species for specific genes by aligning to the guanaco reference gene set using Bowtie2 [72] and then RSEM [73] was used to estimate the Fragments Per Kilobase of transcript per Million mapped reads (FPKM). For the de novo gene predictions, AUGUSTUS (v2.5.5) [74] and GENESCAN (v1.0) [75] were used to identify candidate proteinencoding genes in the masked genome with self-trained model parameters. For homology-based predictions, we used the genomes of B.taurus, C. bactrianus, C. dromedarius, E. caballus, $H$. sapiens, $M$. musculus, and $V$. pacos. These were mapped to a repeat masked assembly using TBLASTN2.2.23 [76] with an E-value threshold of 1E-5. Subsequently, homologous genome sequences were aligned against the matching proteins to define gene models using GENEWISE (v2.2.0) [77]. For the EST-based method, unigenes were mapped to the genome using BLAT (identity $\geq 90 \%$, coverage $\geq 90 \%$ ), and overlaps among the spliced alignments were filtered and linked using PASA [78]. Gene models supported by the three methods were integrated to yield the final geneset using GLEAN (Additional file 1: Table S7a-c). To assign functions to the gene models, we used the SwissProt and TrEMBL protein databases (Uniprot release 201101) using BLASTP with an $E$-value threshold of $\leq 10^{-5}$. Domain-based comparisons were performed and searched to identify conserved domains/families. Functional annotation was carried out using Blast2GO [79]. Metabolic pathway annotations were performed by sequence comparisons with KEGG proteins (Release 76) using BLASTP.

\section{Resequencing of individuals of four camelid species}

Resequencing produced 35-49 Gb of raw data per individual. We removed reads with $\geq 10 \%$ unidentified nucleotides, $>10$ nt aligned to the adaptor, with $\leq 10 \%$ mismatches allowed, with $>50 \%$ bases having phred quality $<5$ and putative PCR duplicates generated during library construction. Details of the throughput and read lengths are summarized in Additional file 1: Table S9.

\section{Chromosome level reference genome and SNP calling}

To obtain the chromosome level reference genome, we aligned our assembled guanaco scaffolds against the VicPac3.1 [80] using LAST [81] and get the chromosome information for each scaffold. Due to the lack of ordering information for scaffolds within each chromosome of their assembly, we joined our scaffolds together after sorted them by length within each VicPac3.1 chromosome. For unmapped scaffolds, we connected them together and classified them as "ChrUN". In total, $~ 2.1 \mathrm{~Gb}$ of assembled scaffolds in our study could be mapped to chromosomes in VicPac3.1 and $\sim 499.7 \mathrm{Mb}$ were classified as "ChrUN," the same level as those reported by VicPac3.1. We also mapped the reads of two selected individuals (one male and one female) onto our assembled scaffolds and identified $X$ fragments based on the sequence depth ratio (male/female $\geq 0.5$ ). We next connected the identified $\mathrm{X}$ fragments together and used the assembled sequence as our $\mathrm{X}$ chromosome sequence.

High-quality reads were then aligned to the above reference genome using BurrowsWheeler Alignment MEM (BWA-MEM) [82], and alignment statistics were obtained using SAMtools (v1.2) [83]; reads were filtered with a map quality lower than 5 and a mismatch longer than $5 \mathrm{bp}$. The reads of female individuals from our study were 
mapped onto the $\mathrm{X}$ chromosome respectively. Base Quality Score recalibration and indel realignment were performed prior to variant calling, which was carried out using Picard (http://broadinstitute.github.io/picard). BAM files were adjusted using the Genome Analysis Toolkit (GATK, v3.4) [84] with the HaplotypeCaller method, using a hidden Markov model likelihood function.

\section{Phylogenetic, admixture, and lineage sorting analyses}

To examine the evolutionary relationships among SACs across the genome, a sliding window with length and step of $250 \mathrm{bp}$ was used to select SNPs, mapped to the guanaco reference genome. One SNP was selected per window, resulting in 5,901,447 positions being analyzed. A neighbor-joining tree (sample location in Fig. 1a) was then constructed using uncorrected P-distances in TreeBestv1.9.2 [85] with 100 bootstraps (Fig. 1a). We also performed population clustering analyses using the software package ADMIXTURE [86]. Specifically, we used PLINK [87] to extract variant sites that were not in significant linkage disequilibrium (LD) with any other site within a $100 \mathrm{~kb}$ window. We then ran ADMIXTURE with $k=2,3$, and 4 (Fig. 1c). We explored the detected admixture further in a phylogenetic context by using Treemix [88], which allows the evaluation of the effects of genetic drift and gene flow on a maximum likelihood phylogeny. This software models the relationship among the sample populations with their ancestral population using genome-wide allele frequency data and a Gaussian approximation of genetic drift. The $f$ index representing the fraction of the variance in the sample covariance matrix $(\hat{w})$ accounted for by the model covariance matrix $(w)$ was used to identify the information contribution of each migration vector added to the tree. Up to 20 possible migration vertices were computed.

We more explored introgression among SAC species in more detail at the genomic level, first by using an ABBA/BABA approach [89] (see Additional file 1: Supplementary Text). The Bactrian camel was used as an outgroup, and all possible four-taxon topologies of alpaca, vicuña, llama, guanaco were analyzed. We calculated both Patterson's $D[90,91]$ and modified $f$ statistics $\left(f_{\mathrm{d}}\right)$ [89]. The frequency of the derived allele on each locus in each species was used instead of binary counts of fixed ABBA and BABA. A significant positive $D$ value signifies an excess of shared derived alleles between taxa while a significant negative value indicates the presence of gene flow. A block size of $30 \mathrm{Mb}$ was selected to calculate standard errors of $D$ by bootstrapping. To identify introgressed segments from llama to alpaca, a 100-kb window size was chosen for calculating the $f_{\mathrm{d}}$ across the whole genome (Fig. 2a, b). We separately examined $D$ and $f_{\mathrm{d}}$ across the constructed $\mathrm{X}$-chromosome to detect whether the pattern of admixture differed from the autosomes.

Finally, we used Local Ancestry Inference and estimates of LA tract length to simultaneously estimate admixture times and LA using a Hidden Markov Model [92]. In the absence of a recombination map and chromosome-scale assembly for SACs, we estimated a genome-wide recombination rate by assuming one cross-over per generation per chromosome, obtaining $1.82 \mathrm{e}-8$ crossovers/base pair; owing to the genetic divergence between guanaco and vicuña, we could extract sites where allele frequencies differed by at least $50 \%$ between the ancestral populations. We required that $90 \%$ of samples had a read depth of 10 or more at each site and that successive ancestry 
informative sites be separated by at least $10 \mathrm{~kb}$, in order to reduce the impacts of LD on admixture time estimation, although we note that LD has a minimal impact on these estimates for genetically divergent ancestral populations such as guanaco and vicuña. For all llama and alpaca samples, we imported the filtered read counts from the $V C F$ files as the primary dataset for Local Ancestry Inference. Annotations of inferred introgressed regions were investigated for putative functions of relevance in SACs, other livestock species, and model mammals, including humans. For each domesticated population, we fitted a single pulse admixture model to genome-wide variation data, using 1000 block bootstraps of size 5000 ancestry informative sites. From these analyses, we simultaneously estimated local ancestry proportions and time since admixture, assuming a single pulse, for llama and alpaca (Fig. 2a, b).

Incomplete lineage sorting among phylogenetically related species, such as the vicuña and guanaco, can potentially obscure signatures of introgression between them (or here, between their domestic descendants, alpaca and llama). We compared the estimates of admixture generated above with estimates using ILS analysis with CoalHMM [93], a maximum likelihood approach that allows the simultaneous estimate of ILS, divergence time, and ancestral effective population size. Only alignment blocks with the L. glama scaffold were included, to enrich for syntenic regions and constrain the number of scaffolds to be analyzed-this resulted in 556 syntenic blocks being included. We called hidden states along the genomic alignment with highest posterior probability and computed the proportion of reference genome positions in each state in windows of $10 \mathrm{~kb}, 100 \mathrm{~kb}$, and $1 \mathrm{Mb}$. We only considered windows where more than $30 \%$ was covered by the analyzed alignment.

\section{Demographic history simulation}

We used the sequential Markovian coalescent to evaluate the demographic history of the four SACs, starting with the Pairwise method (PSMC) [94], which reconstructs long-term changes in effective population size over the evolutionary history of the species (here approximately $2 \mathrm{M}$ to $10 \mathrm{~K}$ years ago), assuming a generation time of 5 years and a mutation rate of $7 \mathrm{E}-9$ per site per generation which was used in the reference [8]. Because admixture is known to bias PSMC outputs in favor of detecting demographic declines [95], we removed the admixture tracts identified using LAI (see the "Results" section) before carrying out the simulations. Regions with LAI probability of 0.8 or greater of originating from introgression were removed and the flanking region reconnected where three or more contiguous sites of non-introgressed ancestry. Deintrogressed sequences were then realigned for downstream analysis. We aligned short reads to their respective reference genomes using BWA-MEM (v0.5.9) with the setting aln $-\mathrm{I}-\mathrm{o} 0-\mathrm{l} 31-\mathrm{k} 2-\mathrm{t} 4$ and aligned the SNPs using SAMtools (v0.1.17), dividing them into non-overlapping $100 \mathrm{bp}$ bins, scored as heterozygous if there was a heterozygote in the bin, or as homozygous otherwise. The resultant bin sequences were used as the input for PSMC. Bootstrapping was performed by randomly resampling 100 sequences from the original batch and the reconstructed population history was plotted using gnuplot4.4. Next, we used multi-sample sequential Markovian coalescent (MSMC) [96] to investigate more recent changes (up to $2 \mathrm{~K}$ years ago). SNP VCF files were obtained using GATK and all sites were phased using SHAPEIT2 [97]; mask files 
were constructed using $B A M$ files, created during the SNP calling step. Two models, "fixedRecombination" and "skipAmbiguous," were used to infer population size changes and divergences. For bootstrapping, pairs of samples within-population and crosspopulation were randomly selected to infer their population size and coalescence rates. MSMC was run with the default parameters and fixed recombination rate for $20 \mathrm{M}$ iterations. Results were plotted in $R$ using ggplot 2 and scaled with a generation time of 5 years and the mutation of $0.7 \mathrm{E}-8$ per site per year. To better understand very recent trends in effective population size for all species (i.e., within the last c1,000 years), we implemented the linkage disequilibrium-based method of SNeP v1.11 [35] that uses high density genome-wide data and can take account of variation in sample size, mutation, phasing, and recombination rate. Using this approach, we further documented short-term changes in Ne by implementing NeS [36], which records the change in slope of the inferred $\mathrm{Ne}$ trend obtained from $\mathrm{SNeP}$, a more detailed picture of population changes over the period studied. A constant rate of change is shown as a flat line proximal to 0 in the $Y$-axis, whereas deviations above and below 0 represent relative increases and reductions in $\mathrm{Ne}$, respectively. Alpaca genomes were analyzed after removal of regions of high guanaco ancestry, retaining $64 \%$ of the original genome, and a more stringent approach was taken of only retaining the regions with high vicuña ancestry, retaining $43 \%$ of the original genome.

\section{Selection signatures of environmental adaptation, domestication, and introgression}

We took a population genomics approach to identify selection during SAC domestication, by comparing the (ancestral) V.v. mensalis samples with alpaca and the (ancestral) L. g. cacsilensis samples with llama, using the whole-genome datasets where introgressed tracts were removed. First, selection signatures were explored using $F_{\mathrm{ST}}$ outliers, comparing them to the null distribution generated in windows of $100 \mathrm{~kb}$, using VCFtools [28]. We used a windowed $F_{\mathrm{ST}}$ as a test statistic, retaining windows with values exceeding the $99 \%$ upper quantile as potential locations for selection. Since $F_{\mathrm{ST}}$ analysis does not differentiate between ancestor or domestic signatures of selection, we also used Selscan 1.1.0b [98] to implement extended haplotype homozygosity analysis (XP-EHH). XP-EHH scores were standardized across the genome and those exceeding the top $1 \%$ of the distribution were identified as potential signals of positive selection. Contiguous significant SNPs were integrated into a common signature or region within each comparison, allowing for one non-significant SNP and including half of the physical distance to the neighboring non-significant marker on both sides. Since XP-EHH searches for unusually long haplotypes, isolated significant SNPs were discarded. Positively selected regions were examined for the presence of genes of known adaptive significance first in llama and alpaca, then using the literature for other livestock species, and finally for evidence from model mammal species and humans. Finally, to search for putatively adaptively introgressed segments of non-native ancestry in the genomes of alpaca and llama, we compared the results of the selection signature analysis with the introgression analysis to look for common signatures and searched the genomes for sites where all sequenced alpacas and llamas were homozygous for introgressed guanaco or vicuña-like ancestry, respectively. We required that at least four consecutive markers support a region as introgressed. We then investigated fine-scale patterns of 
DNA variation in four regions containing the ANTXR2/PRDM8/FGF5/C4orf22, EDN3, OR5 family, and OR2 family genes to compare sequence variation in vicuña, guanaco, llama, and alpaca to both verify the ancestry of these segments and to examine $F_{\mathrm{ST}}$ distribution along the segments when comparing llama and alpaca. This was carried out to localize the regions of the gene responsible for the high overall $F_{\mathrm{ST}}$ and LAI scores and to examine their distribution to infer locus-specific selection processes in these genes.

\section{Functional analysis of target genes}

To further investigate potential function of genes identified in the selection signature analysis, we first focused on a gene within our top ranking admixed locus (in the direction of llama to alpaca), comprising a set of candidate genes for adaptive introgression and artificial selection (ANTXR2/PRDM8/FGF5/C4orf22; see the "Results" section) [99, $100]$ and in particular on FGF5. Since functional variation at FGF5 (also known as the angora gene) is known to correlate with short- or longwool phenotypes in sheep and goat [101, 102], we examined sequence differences in this gene in short and longwool alpaca. Six healthy 2 year-old longwool (Suri) alpacas (3:3) and shortwool (Huacaya) alpacas (3:3) were selected for mRNA and protein expression testing using the skin samples. A skin section (diameter $3 \mathrm{~cm}$ ) from the neck was surgically obtained and immediately stored in liquid nitrogen. For mRNA PCR and qPCR, $1 \mu$ g of total RNA from Suri and Huacaya alpaca skins was extracted using TRIzol (Invitrogen) and treated with DNase I (Sigma). The mRNA was converted to cDNA using a cDNA synthesis kit (Takara, Dalian, China) for PCR and qPCR using SYBR Green (Takara) on a 7500 Fast Real-Time PCR system (Applied Biosystems) using FGF5 primers (Additional file 1: Table S21). The PCR reaction is conducted as follows: $95^{\circ} \mathrm{C}$ for $5 \mathrm{~min}$, followed by 40 cycles of $95^{\circ} \mathrm{C} 15 \mathrm{~s}, 56^{\circ} \mathrm{C}$ or $58^{\circ} \mathrm{C}$ for $30 \mathrm{~s}$, and $72{ }^{\circ} \mathrm{C}$ for $30 \mathrm{~s}$. PCR products were sequenced using standard Sanger methods and analyzed using an ABI 3730XL semi-automatic DNA analyzer. For Western Blot analysis, protein samples were separated using 10\% SDS-PAGE and transferred to PVDF membranes. The membranes were blocked with $5 \%$ skimmed milk for $2 \mathrm{~h}$ and incubated for overnight at $4{ }^{\circ} \mathrm{C}$ with the diluted primary rabbit antibodies FGF5 (1:500) and $\beta$-actin (1:1000). After washing four times for $5 \mathrm{~min}$ each with TBST, the membranes were incubated for $1 \mathrm{~h}$ at $37^{\circ} \mathrm{C}$ with horseradish peroxidase (HRP) secondary antibodies against rabbit IgG (1: 10000). After washing four times for $5 \mathrm{~min}$ each with TBST, the bound antibodies were visualized by chemiluminescence using ECL. Immunoblots were scanned on a Chemi$\mathrm{DOC}^{\mathrm{m}} \mathrm{XRS}+$ imager (Bio-Rad), and protein levels were quantified using Image-Pro Plus (Olympus). Finally, we examined the expression profiles (FPKM) of 20 single copy genes identified in the introgression and selection analysis (see above; Additional file 1: Table S22). Comparative inference from expression profiles was limited because of different tissue source (skin samples from 17 alpacas from China and blood samples from each of the de novo sequenced vicuña, guanaco, and llama).

To investigate the potential effect of hypoxia on the expression of the HSA4q21 syntenic locus (ANTXR2/PRDM8/FGF5/C4orf22), alpaca melanocytes and sheep melanocytes were cultured for $72 \mathrm{~h}$ under normoxic $\left(21 \% \mathrm{O}_{2}\right)$ and hypoxic $\left(\begin{array}{lll}13 \% & \mathrm{O}_{2}\end{array}\right)$ conditions respectively. Alpaca melanocytes and sheep melanocytes were lysed to get protein by RIPA (Beyotime, Shanghai, China) for Western Blot analysis with the diluted 
primary rabbit antibodies FGF5, ANTXR2, PRDM8, C4orf22 (all at a ratio of 1:500), and $\beta$-actin (1:1000) using the same protocol above described. Total RNA of alpaca melanocytes was extracted by Trizol (Invitrogen, Carlsbad, CA, USA) for qPCR analysis of SOX6 and HIF1 $\alpha$ (the primers were listed in Table S21).

\title{
Supplementary information
}

Supplementary information accompanies this paper at https://doi.org/10.1186/s13059-020-02080-6.

Additional file 1: Figure S1. K-mer distribution. Figure S2. Detailed sampling map of all sequenced individual. Figure S3. Treemix phylogeny and variance plot. Figure S4. Introgression segments into llama $\left(f_{\mathrm{d}}\right)$. Figure S5. PSMC plot for all four SAC species. Figure S6. MSMC plots for (a) guanaco; (b) llama; (c) vicuña; (d) alpaca. Figure S7. Manhattan plot of selection signatures detected in the comparison between vicuña and alpaca (XP-EHH upper FST bottom). Figure S8. Manhattan plot of selection signatures detected in the comparison between guanaco and llama (XP-EHH upper, FST bottom). Figure S9. Comparison between region-wide FST and SNP distribution among wild ancestors and domestic relatives for the OR5 and OR2 olfactory receptor family. Figure S10. Comparison between region-wide FST and SNP distribution among wild ancestors and domestic relatives for the HoxD gene clusters and OLA1 gene related to morphology development. Figure S11. SNP distribution among wild ancestors and domestic relatives with evidence of introgression from llama to alpaca on Chr10. Figure S12. SNP distribution among wild ancestors and domestic relatives with evidence of introgression from alpaca to llama: CORIN, FGF21, GNAS, TAGLN. Figure S13. cDNA sequence, expression histogram, Western Blot and protein expression histogram for the alpaca skin FGF5 expression analysis. Figure S14. The phylogeny of South American camelids with segments of high guanaco ancestry removed from alpaca genomes. Table S1. Statistics of the clean data for the de novo genomes. Table S2a-c. Assembly statistics for each species. Table S3. Genome coverage assessed by transcriptome unigenes. Table S4. The BUSCO results of the three new assembled SAC genomes. Table S5. K-mer analysis. Table S6. Aligned sequence data for de novo genomes. Table S6a. Aligned sequence between the de novo genomes and related species: pairwise whole-genome alignment was performed using LASTZ. Table S6b. Synteny analysis for aligned de novo genomes. Table S7a-c. the number of predicted genes for each species. Table S8. Sampling details. Table S9. Resequencing data summary. Table S10. ABBA-BABA statistics. Table S11. Introgressed segments from llama into alpaca using fd and LAl. Table S12. Introgressed segments from alpaca into llama using fd and LAI. Table S13. Introgressed segment and genes showing low and high LAl introgression on the inferred X-chromosome of alpaca. Table S14. Selection signatures detected in comparisons between vicuña and alpaca (XP-EHH). Table S15. Selection signatures detected in comparisons between vicuña and alpaca (FST). Table S16. Selection signatures detected in comparisons between vicuña and alpaca (overlap between methods). Table S17. Selection signatures detected in comparisons between guanaco and llama (XP-EHH). Table S18. Selection signatures detected in comparisons between guanaco and llama (FST). Table S19. Selection signatures detected in comparisons between guanaco and llama (overlap between methods). Table S20. Olfactory receptor gene numbers in South American camelids, compared to the cow. Table S21. PCR and qPCR primers. Table S22. Blood and skin tissue expression counts for de novo sequenced SACs and Chinese alpaca, respectively. Supplementary Note S1 - De novo Genome Sequencing a. Genome size estimation. b. De novo sequencing, assembly and annotation. Supplementary Note S2 - Introgression analysis. Supplementary Note S3- Sample collection, DNA extraction and permits.

Additional file 2. Review history.

\begin{abstract}
Abbreviations
AlpGua: Alpaca genome that removed guanaco ancestry sites; AlpVic: Alpaca genome that only remain vicuña ancestry sites; ASIP: Agouti signaling protein; ANTXR: Anthrax toxin receptor; CORIN: Atrial natriuretic peptideconverting enzyme; BAM: Binary Alignment/Map; BP: Before present; BUSCO: Benchmarking Universal Single-Copy Orthologs; BLAT: BLAST-like Alignment Tool; BWA-MEM: Burrows-Wheeler Alignment MEM; CSTZ: Cathepsin Z; CDS: Coding sequence; C4orf22: Chromosome 4 open reading frame 22; ECL: Electrochemiluminescence; XPEHH: Extended haplotype homozygosity; EDN3: Endothelin 3; FGF: Fibroblast growth factor; FPKM: Fragments Per Kilobase of transcript per Million mapped reads; GATK: Genome Analysis Toolkit; GO: Gene Ontology; HRP: Horseradish peroxidase; HIF: Hypoxia-inducible factor; PHD: Prolyl hydroxylase domain; SOX: Sry-related HMG-box-containing transcription factor; KEGG: Kyoto Encyclopedia of Genes and Genomes; LD: Linkage disequilibrium; ILS: Incomplete lineage sorting; LAl: Local Ancestry Inference; $f_{\mathrm{d}}$ : Modified $f$ statistics; MYA: Millions of years ago; MSMC: Multiple Sequence Markovian Coalescent; OR: Olfactory receptor; OLA1: Obg-like ATPase 1; PSMC: Pairwise Sequential Markovian Coalescent; PVDF: Polyvinylidene fluoride; PRDM8: PR domain containing 8; qPCR: Quantitative real-time PCR; QTL: Quantitative trait loci; SAMtools: Sequence Alignment/Map (SAM) tools; SNP: Single-nucleotide polymorphism; SACs: South American camelids; SNeP: SNP linkage disequilibrium-based Ne estimation; TAGLN: Transgelin; VCFtools: Variant call format tools; GWAS: Whole-genome association studies
\end{abstract}

\section{Acknowledgements}

We thank Mr. Tianyong Liu for the help during the sample collection. 
Peer review information

Tim Sands was the primary editor of this article and managed its editorial process and peer review in collaboration with the rest of the editorial team.

\section{Authors' contributions}

C.D. conceived the project and coordinated the de novo genome sequencing. R.F., Z.G., X.G., and J.C.M. designed and performed experiments. V.V., B.G., Y.H., E.L., X.S., X.Y., C.Z., W.G., J.H., K.M., R.C., X.Z., and M.B. provided input on experimental and study design. X.G., Z.G., M.W.B., S.P., and X.Z. coordinated the data analysis. M.W.B. wrote the manuscript with input from R.F., Z.G., X.G., J.C.W., X.Z., and C.D. X.Z., and M.W.B. supervised the project.

\section{Funding}

This work is funded by the projects of Agro-Scientific Research in the Public Interest of China (No. 201303119), the Strategic Priority Program of the Chinese Academy of Sciences (XDB31000000), National Natural Science Foundation of China (31821001), Cardiff University and the Chinese Academy of Sciences President's International Fellowship Initiative, the Young Sanjin Scholars Distinguished Professor program and the Aid Program for Innovation Research Team in Shanxi Agricultural University (CXTD201201), FONDECYT grant (1140785) CONICYT of Chile, Science and Technology Department of Qinghai Province Major Project "Sanjiangyaun National Park Animal Genome Program," and Second Tibetan Plateau Scientific Expedition and Research Program (STEP) (No. 2019QZKK0501).

\section{Availability of data and materials}

The genome assembly generated and analyzed during the current study are available in the NCBI under BioProject ID PRJNA427832 [103], PRJNA421373 [104], and PRJNA427644 [105]. The SAC whole-genome shotgun project has been deposited at the GenBank under the accession number PRJNA612032 [106]. The RNA-seq data of Alpaca could be accessed through PRJNA636766 [107]. Other results are included in this published article and its Additional material files.

\section{Ethics approval and consent to participate}

All animals were handled and treated according to the alpaca guidelines approved by the Animal Ethics Committee [2017(55)] at Shanxi Agricultural University (Taigu, Shanxi, China).

\section{Consent for publication}

Not applicable.

\section{Competing interests}

The authors declare that they have no competing interests.

\section{Author details}

${ }^{1}$ College of Animal Science and Veterinary Medicine, Shanxi Agricultural University, Taigu, Shanxi, China. ${ }^{2}$ CAS Key Lab of Animal Ecology and Conservation Biology, Institute of Zoology, Chinese Academy of Sciences, Beijing, China. ${ }^{3}$ Cardiff University - Institute of Zoology Joint Laboratory for Biocomplexity Research, Chinese Academy of Sciences, Beijing, China. ${ }^{4}$ University of Chinese Academy of Sciences, Beijing, China. ${ }^{5}$ BGl Genomics, BGl, Shenzhen, China. ${ }^{6}$ Departamento de Ciencias Básicas, Facultad de Ciencias, Universidad del Bio Bio, Chillán, Chile. ${ }^{7}$ Programa de Doctorado en Ciencias mención Ecología y Evolución, Escuela de Graduados, Facultad de Ciencias., Universidad Austral de Chile, Valdivia, Chile. ${ }^{8}$ Facultad de Ciencias Forestales y de la Conservación de la Naturaleza, Universidad de Chile, Santiago, Chile. ${ }^{9}$ CONOPA-Instituto de Investigación y Desarrollo de Camélidos Sudamericanos, Pachacamac, Lima, Peru. ${ }^{10}$ Bioinformatics Research Centre, Aarhus University, Aarhus, Denmark. ${ }^{11}$ Department of Biomolecular Engineering and Genomics Institute, UC Santa Cruz, Santa Cruz, CA, USA. ${ }^{12}$ Department of Animal Science, Food and Technology DIANA, Università Cattolica del Sacro Cuore, Piacenza, Italy. ${ }^{13}$ Center for Excellence in Animal Evolution and Genetics, Chinese Academy of Sciences, Kunming, China. ${ }^{14}$ School of Biosciences and Sustainable Places Institute, Cardiff University, Cardiff, Wales, UK.

Received: 8 October 2019 Accepted: 21 June 2020

Published online: 02 July 2020

\section{References}

1. Zeder M. Central questions in the domestication of plants and animals. Evol Anthropol Issues News Rev. 2006;15:105-17.

2. Gade DW. Carl Troll on Nature and Culture in the Andes (Carl Troll über die Natur und Kultur in den Anden). Erdkunde. 1996:50:301-16.

3. Wheeler JC, Russel AJF, Redden H. Llamas and alpacas: pre-conquest breeds and post-conquest hybrids. J Archaeol Sci. 1995:22:833-40.

4. Kadwell M, Fernandez M, Stanley HF, Baldi R, Wheeler JC, Rosadio R, et al. Genetic analysis reveals the wild ancestors of the llama and the alpaca. Proc Biol Sci. 2001;268:2575-84.

5. Gentry A, Clutton-Brock J, Groves CP. The naming of wild animal species and their domestic derivatives. J Archaeol Sci. 2004:31:645-51.

6. Gonalons GLM. Camelids in ancient Andean societies : a review of the zooarchaeological evidence. Quat Int. 2008;185: 59-68.

7. Marín JC, Romero K, Rivera R, Johnson WE, Gonzalez BA. Y-chromosome and mtDNA variation confirms independent domestications and directional hybridization in South American camelids. Anim Genet. 2017;48:591-5.

8. Wu H, Guang X, Al-Fageeh MB, Cao J, Pan S, Zhou H, et al. Camelid genomes reveal evolution and adaptation to desert environments. Nat Commun. 2014;5:5188.

9. Luo R, Liu B, Xie Y, Li Z, Huang W, Yuan J, et al. SOAPdenovo2: an empirically improved memory-efficient short-read de novo assembler. Gigascience. 2012;1:18. 
10. Parra G, Bradnam K, Korf BI. CEGMA: a pipeline to accurately annotate core genes in eukaryotic genomes. Bioinformatics. 2007;23:1061-7.

11. Haubold B, Wiehe T. How repetitive are genomes? BMC Bioinformatics. 2006;7:1-10.

12. Ammann C, Jenny B, Kammer K, Messerli B. Late Quaternary Glacier response to humidity changes in the arid Andes of Chile (18-295). Palaeogeogr Palaeoclimatol Palaeoecol. 2001;172:313-26.

13. Marín JC, Casey CS, Kadwell M, Yaya K, Hoces D, Olazabal J, et al. Mitochondrial phylogeography and demographic history of the vicuña: implications for conservation. Heredity. 2007;99:70-80.

14. Niimura Y, Matsui A, Touhara K. Extreme expansion of the olfactory receptor gene repertoire in African elephants and evolutionary dynamics of orthologous gene groups in 13 placental mammals. Genome Res. 2014;24:1485-96.

15. Moayeri M, Leysath CE, Tremblay JM, Vrentas C, Crown D, Leppla SH, et al. A heterodimer of a VHH (variable domains of camelid heavy chain-only) antibody that inhibits anthrax toxin cell binding linked to a VHH antibody that blocks oligomer formation is highly protective in an anthrax spore challenge model. J Biol Chem. 2015;290:6584-95.

16. Daverio MS, Vidal-Rioja L, Frank EN, Di Rocco F. Molecular characterization of the llama FGF5 gene and identification of putative loss of function mutations. Anim Genet. 2017;48:716-9.

17. Park SY, Lee HJ, Ji SM, Kim ME, Jigden B, Lim JE, et al. ANTXR2 is a potential causative gene in the genome-wide association study of the blood pressure locus 4q21. Hypertens Res. 2014;37:811-7.

18. Shin $Y$, Jung $H$, Jung $M$, Yoo $S$, Subramaniyam $S$, Markkandan $K$, et al. Discovery of gene sources for economic traits in hanwoo by whole-genome resequencing. Asian-Australas J Anim Sci. 2016;29:1353-62.

19. Segun-Busari $\mathrm{S}$, Ayodele $\mathrm{O}$, Dunmade AD, Afolabi O, Omokanye H, Owoeye JF. Blue eyes syndrome and deafness: the need for early audiological screening children. Nig J Otorhinolaryngol. 2018;16(2):1-3.

20. Wang R, Chen T, Zhao B, Fan R, Ji K, Yu X, et al. FGF21 regulates melanogenesis in alpaca melanocytes via ERK1/2mediated MITF downregulation. Biochem Bioph Res Co. 2017;490(2):466-71.

21. Feeley NL, Bottomley S, Munyard KA. Three novel mutations in ASIP associated with black fibre in alpacas (Vicugna pacos). J Agr Sci. 2011;149:529-38.

22. Silvana Daverio M, Rigalt F, Romero S, Vidal-Rioja L, Di Rocco F. Polymorphisms in MCIR and ASIP genes and their association with coat color phenotypes in llamas (Lama glama). Small Ruminant Res. 2016;144:83-9.

23. Norris BJ, Bower NI, Smith WJM, Cam GR, Reverter A. Gene expression profiling of ovine skin and wool follicle development using a combined ovine-bovine skin CDNA microarray. Aust J Exp Agric. 2005;45:867-77.

24. Sikora KM, Magee DA, Berkowicz EW, Berry DP, Howard DJ, Mullen MP, et al. DNA sequence polymorphisms within the bovine guanine nucleotide-binding protein Gs subunit alpha (Gsa)-encoding (GNAS) genomic imprinting domain are associated with performance traits. BMC Genet. 2011;12:4-19.

25. Sabine K, Bernhardt A, Kalinski T, Baldensperger M, Zeh M, Teller A, et al. Induction of premalignant host responses by cathepsin X/Z-deficiency in helicobacter pylori-infected mice. PLoS One. 2013;8:e70242.

26. Neyra V, Chavarry E, Espinoza JR. Cysteine proteinases Fas1 and Fas2 are diagnostic markers for Fasciola hepatica infection in alpacas (Lama pacos). Vet Parasitol. 2002;105:21-32.

27. Haase E. Comparison of reproductive biological parameters in male wolves and domestic dogs. Zeitschrift Fü Saugetierkunde. 2000;65:257-70

28. Danecek P, Auton A, Abecasis G, Albers CA, Banks E, DePristo MA, et al. The variant call format and VCFtools. Bioinformatics. 2011:27:2156-8.

29. Noor A, Whibley A, Marshall CR, Gianakopoulos PJ, Piton A, Carson AR, et al. Disruption at the PTCHD1 locus on Xp22. 11 in autism spectrum disorder and intellectual disability. Sci Transl Med. 2010;2:49-68.

30. Torrico B, Fernàndez-Castillo N, Hervás A, Milà M, Salgado M, Rueda I, et al. Contribution of common and rare variants of the PTCHD1 gene to autism spectrum disorders and intellectual disability. Eur J Hum Genet. 2015;23:1694-701.

31. Ung DC, lacono G, Méziane H, Blanchard E, Papon MA, Selten M, et al. PTCHD1 deficiency induces excitatory synaptic and cognitive dysfunctions in mouse. Mol Psychiatry. 2018;23:1356-67.

32. Franklin WL. Contrasting socioecologies of South America's wild camelids: the vicuña and the guanaco. Advances in the study of mammalian behavior (JF Eisenberg and DG Kleiman, eds.). Special Publication. 1983;7:573-629.

33. Pacifici M, Santini L, Marco MD, Baisero D, Francucci L, Marasini GG, et al. Generation length for mammals. Nature Conserv. 2013;5:87-94.

34. Setchell BP. Domestication and reproduction. Anim Reprod Sci. 1992;8:195-202.

35. Barbato M, Orozco-Terwengel P, Tapio M, Bruford MW. SNeP: a tool to estimate trends in recent effective population size trajectories using genome-wide SNP data. Front Genet. 2015;6:109.

36. Pitt D, Bruford MV, Barbato M, Orozco-Terwengel P, Martínez R, Sevane N. Demography and rapid local adaptation shape Creole cattle genome diversity in the tropics. Evol Appl. 2019;12:105-22.

37. Matthews D, Diskin MG, Kenney DA, Creevey C, Keogh K, Wsters S. Effect of short term diet restriction on gene expression in the bovine hypothalamus using next generation RNA sequencing technology. BMC Genomics. 2017;18: 857-70.

38. Zhang FP, Poutanen M, Wilbertz J, Huhtaniemi I. Normal prenatal but arrested postnatal sexual development of luteinizing hormone receptor knockout (LuRKO) mice. Mol Endocrinol. 2002;15:172-83.

39. Ozawa K, Kuwabara K, Tamatani M, Takatsuji K, Tsukamoto Y, Kaneda S, et al. 150-kDa oxygen-regulated protein (ORP150) suppresses hypoxia-induced apoptotic cell death. J Biol Chem. 1999;274:6397-404.

40. Piórkowska K, Żukowski K, Ropka-Molik K, Tyra M. Detection of genetic variants between different Polish Landrace and Puławska pigs by means of RNA-seq analysis. Anim Genet. 2018:49:215-25.

41. Cao $Y$, Jin HG, Ma HH, Zhao Z. Comparative analysis on genome-wide DNA methylation in longissimus dorsi muscle between Small Tailed Han and Dorper × Small Tailed Han crossbred sheep. Asian-Australas J Anim Sci. 2017;30:1529-39.

42. Pérez O'Brien AM, Utsunomiya YT, Mészáros G, Bickhart DM, Liu GE, Tassell CPV, et al. Assessing signatures of selection through variation in linkage disequilibrium between taurine and indicine cattle. Genet Sel Evol. 2014;46:19-32.

43. Mei C, Wang H, Liao Q, Wang L, Cheng G, Wang H, et al. Genetic architecture and selection of Chinese cattle revealed by whole genome resequencing. Mol Biol Evol. 2017;35:688-99.

44. Park SY, Walker JJ, Johnson NW, Zhao Z, Lightman SL, Spiga F. Constant light disrupts the circadian rhythm of steroidogenic proteins in the rat adrenal gland. Mol Cell Endocrinol. 2013;371:114-23. 
45. Guridi M, Soret B, Alfonso L, Arana A. Single nucleotide polymorphisms in the Melanocortin 1 Receptor gene are linked with lightness of fibre colour in Peruvian alpaca (Vicugna pacos). Anim Genet. 2011;42:679-82.

46. Hadjimarkou MM, Vasudevan N. GPER1/GPR30 in the brain: crosstalk with classical estrogen receptors and implications for behavior. J Steroid Biochem Mol Biol. 2018;176:57-64.

47. Peng Y, Liu X, Geng L, Ma R, Li L, Li J, et al. Illumina-sequencing based transcriptome study of coat color phenotypes in domestic goats. Genes Genom. 2017;39:817-30.

48. Jackling FC, Johnson WE, Appleton BR. The genetic inheritance of the blue-eyed white phenotype in alpacas (Vicugna pacos). J Hered. 2014;105:847-57.

49. Kaelin CB, Xu X, Hong LZ, David VA, McGowan KA, Schmidt-Küntzel A, et al. Specifying and sustaining pigmentation patterns in domestic and wild cats. Science. 2012;337:1536-41.

50. Strain GM. The genetics of deafness in domestic animals. Front Vet Sci. 2015;2:1-20.

51. Hérault $Y$, Fraudeau N, Zákány J, Duboule D. Ulnaless (UI): a regulatory mutation inducing both loss-of-function and gain-of-function of posterior HoxD genes. Development. 1997;124:3493-500.

52. Ding Z, Liu Y, Rubio V, He J, Minze LJ, Shi ZZ. OLA1, a translational regulator of p21, maintains optimal cell proliferation necessary for developmental progression. Mol Cell Biol. 2016;36:2568-82.

53. Dong CS. Biology of the alpaca. Beijing: China Book Press; 2015.

54. Witt KE, Huerta-Sánchez E. Convergent evolution in human and domesticate adaptation to high-altitude environments. Philos T R Soc B. 2019;374:1777.

55. Lin SC, Lee HC, Hsu CT, Huang YH, Li WN, Hsu PL, et al. Targeting anthrax toxin receptor 2 ameliorates endometriosis progression. Theranostics. 2019;9:620-32.

56. Khan WS, Adetola BA, Imothy EH. Hypoxic conditions increase hypoxia-inducible transcription factor $2 a$ and enhance chondrogenesis in stem cells from the infrapatellar fat pad of osteoarthritis patients. Arthritis Res Ther. 2007;9:R55.

57. Simon G. Population genetics models of local ancestry. Genetics. 2012;191:607-19.

58. Casey CS, Orozco-terWengel P, Yaya K, Kadwell M, Fernández M, Marín JC, et al. Comparing genetic diversity and demographic history in co-distributed wild South American camelids. Heredity. 2018;121:387-400.

59. Hughjones ME, De W. Anthrax and wildlife. Rev Sci Tech. 2002;21:359-83.

60. Zhang Q, Calus MP, Bosse M, Sahana G, Lund MS, Guldbrandtsen B. Human-mediated introgression of haplotypes in a modern dairy cattle breed. Genetics. 2018;209:1305-17.

61. Xi B, Shen Y, Zhao X, Chandak GR, Cheng H, Hou D, et al. Association of common variants near six genes (ATP2B1, CSK, MTHFR, CYP17A1, STK39 and FGF5) with blood pressure hypertension risk in Chinese children. J Hum Hypertension. 2014;28:32-6.

62. Mingji C, Onakpoya IJ, Perera R, Ward AM, Heneghan CJ. Relationship between altitude and the prevalence of hypertension in Tibet: a systematic review. Heart. 2015;101:1054-60.

63. Huerta-Sánchez E, Jin X, Bianba AZ, Peter BM, Vinckenbosch N, Liang Y, et al. Altitude adaptation in Tibetans caused by introgression of Denisovan-like DNA. Nature. 2014;512:194-7.

64. Allen K. Identification of downstream targets of the putative transcription factor PRDM8. Master's Thesis. Toronto: University of Toronto; 2008.

65. Ke Q, Costa M. Hypoxia-inducible factor-1 (HIF-1). Mol Pharmacol. 2006;70(5):1469-80,

66. Zhan X, Culpepper A, Reddy M, Loveless J, Goldfarb M. Human oncogenes detected by a defined medium culture assay. Oncogene. 1987;1:369-76.

67. Ghassemi S, Vejdovszky K, Sahin E, Ratzinger L, Schelch K, Mohr T, et al. Fgf5 is expressed in melanoma and enhances malignancy in vitro and in vivo. Oncotarget. 2017;8(50):87750-62.

68. Seo HR, Jeong HE, Joo HJ, Choi SC, Park CY, Kim JH, et al. Intrinsic FGF2 and FGF5 promotes angiogenesis of human aortic endothelial cells in 3D microfluidic angiogenesis system. Sci Rep. 2016;6:28832.

69. Higgins CA, Petukhova L, Harel S, Ho YY, Drill E, Shapiro L, et al. FGF5 is a crucial regulator of hair length in humans. Proc Natl Acad Sci U S A. 2014;111(29):10648-53.

70. Chan SF, Huang X, McKercher SR, Zaidi R, Okamoto SI, Nakanishi N, et al. Transcriptional profiling of MEF2-regulated genes in human neural progenitor cells derived from embryonic stem cells. Genomics Data. 2015;3:24-7.

71. Pallotti S, Pediconi D, Subramanian D, Molina MG, Antonini M, Morelli MB, et al. Evidence of post-transcriptional read through regulation in FGF5 gene of alpaca. Gene. 2018;647:121-8.

72. Langmead B, Salzberg SL. Fast gapped-read alignment with Bowtie 2. Nat Methods. 2012;9:357-9.

73. Bo L, Dewey CN. RSEM: accurate transcript quantification from RNA-Seq data with or without a reference genome. BMC Bioinformatics. 2011;12:323-38.

74. Mario S, Burkhard M. AUGUSTUS: a web server for gene prediction in eukaryotes that allows user-defined constraints. Nucleic Acids Res. 2005;33:W465-7.

75. Burge C, Karlin S. Prediction of complete gene structure in human genomic DNA. J Mol Biol. 1997;268:78-94.

76. Altschul SF, Madden TL, Schäffer AA, Zhang J, Zhang Z, Miller W, et al. Gapped BLAST and PSI-BLAST: a new generation of protein database search programs. Nucleic Acids Res. 1997;25:3389-402.

77. Birney E, Clamp M, Durbin R. GeneWise and Genomewise. Genome Res. 2004;14:988-95.

78. Haas BJ, Delcher AL, Mount SM, Wortman JR, White O. Improving the Arabidopsis genome annotation using maximal transcript alignment assemblies. Nucleic Acids Res. 2003;31:5654-66.

79. Conesa A, Götz S, García-Gómez JM, Terol J, Talón M, Robles M. Blast2GO: a universal tool for annotation, visualization and analysis in functional genomics research. Bioinformatics. 2005;21:3674-6.

80. Richardson MF, Munyard KA, Croft $L$, Allnutt TR, Jackling F, Alshanbari F, et al. Chromosome-level alpaca reference genome VicPac 3.1 improves genomic insight into the biology of New World camelids. Frontiers Genet. 2019;10:586

81. Kiełbasa SM, Wan R, Sato K, Horton P, Frith MC. Adaptive seeds tame genomic sequence comparison. Genome Res. 2011;21(3):487-93.

82. Li H, Durbin R. Fast and accurate short read alignment with Burrows-Wheeler transform. Bioinformatics. 2009;25:1754-60.

83. Li H, Handsaker B, Wysoker A, Fennell T, Ruan J, Homer N, et al. The sequence alignment/map format and SAMtools. Bioinformatics. 2009;25:2078-9.

84. McKenna A, Hanna M, Banks E, Sivachenko A, Cibulskis K, Kernytsky A, et al. The Genome Analysis Toolkit: a MapReduce framework for analyzing next-generation DNA sequencing data. Genome Res. 2010;20:1297-303. 
85. Vilella AJ, Severin J, Ureta-Vidal A, Heng L, Durbin R, Birney E. Ensembl Compara GeneTrees: Complete, duplicationaware phylogenetic trees in vertebrates. Genome Res. 2009;19:327-35.

86. Alexander DH, Novembre J, Lange K. Fast model-based estimation of ancestry in unrelated individuals. Genome Res. 2009;19:1655-64.

87. Purcell S, Neale B, Todd-Brown K, Thomas L, Ferreira MAR, Bender D, et al. PLINK: a tool set for whole-genome association and population-based linkage analyses. Am J Hum Genet. 2007;81:559-75.

88. Pickrell JK, Pritchard JK. Inference of population splits and mixtures from genome-wide allele frequency data. PLoS Genet. 2012;8:e1002967.

89. Martin SH, Davey JW, Jiggins CD. Evaluating the use of ABBA-BABA statistics to locate introgressed loci. Mol Biol Evol. 2015;32:244-57.

90. Green RE, Krause J, Briggs A, Maricic T, Stenzel U, Kircher M, et al. A draft sequence of the neandertal genome. Science. 2010;328:710-22.

91. Durand EY, Nick P, David R, Montgomery S. Testing for ancient admixture between closely related populations. Mol Biol Evol. 2011;28:2239-52.

92. Corbett-Detig R, Nielsen RA. Hidden Markov model approach for simultaneously estimating local ancestry and admixture time using next generation sequence data in samples of arbitrary ploidy. PLoS Genet. 2017;13:e1006529.

93. Hobolth A, Dutheil JY, Hawks J, Schierup MH, Mailund T. Incomplete lineage sorting patterns among human, chimpanzee, and orangutan suggest recent orangutan speciation and widespread selection. Genome Res. 2011;21:349-56.

94. Li H, Durbin R. Inference of human population history from individual whole-genome sequences. Nature. 201 1;475:493-6.

95. Orozco-Terwengel PA, Bruford MW. Mixed signals from hybrid genomes. Mol Ecol. 2014;23:3941-3.

96. Schiffels S, Durbin R. Inferring human population size and separation history from multiple genome sequences. Nat Genet. 2014;46:919-25

97. Delaneau O, Marchini J, the 1000 Genomes Project Consortium. Integrating sequence and array data to create an improved 1000 Genomes Project haplotype reference panel. Nat Commun. 2013;5:3934.

98. Szpiech ZA, Hernandez RD. selscan: an efficient multithreaded program to perform EHH-based scans for positive selection. Mol Biol Evol. 2014;31:2824-7.

99. He X, Chao Y, Zhou G, Chen Y. Fibroblast growth factor 5-short (FGF5s) inhibits the activity of FGF5 in primary and secondary hair follicle dermal papilla cells of cashmere goats. Gene. 2016;575:393-8.

100. Wang T, Zhang Y, Wang HD, Shen Y, Liu N, Cao J, et al. Alpaca fiber growth is mediated by microRNA let-7b via downregulation of target gene FGF5. Genet Mol Res. 2015;14:13754-63.

101. Li WR, Liu CX, Zhang XM, Chen L, Peng XR, He SG, et al. CRISPR/Cas9-mediated loss of FGF5 function increases woo staple length in sheep. FEBS J. 2017;284:2764-73.

102. Wang X, Cai B, Zhou J, Zhu H, Niu Y, Ma B, et al. Disruption of FGF5 in cashmere goats using CRISPR/Cas9 results in more secondary hair follicles and longer fibers. PLoS One. 2016;1 1:e0164640.

103. Fan R, Gu Z, Guang X, Marín JC, Varas V, Gonzalez B, Wheeler J, Hu Y, Li E, Sun X, Yang X, Zhang C, Gao W, He J, Munch K, Corbett-Detig R, Barbato M, Pan S, Zhan X, Bruford W M, Dong C. Vicugna vicugna mensalis isolate: CD3-2017 Genome sequencing and assembly. NCBI Bioproject. 2018. https:/www.ncbi.nlm.nih.gov/bioproject/PRJNA427832.(2018-10-1).

104. Fan R, Gu Z, Guang X, Marín JC, Varas V, Gonzalez B, Wheeler J, Hu Y, Li E, Sun X, Yang X, Zhang C, Gao W, He J, Munch K, Corbett-Detig R, Barbato M, Pan S, Zhan X, Bruford W M, Dong C. Lama glama chaku isolate: CD1-2017 Genome sequencing and assembly. NCBI Bioproject. 2018. https://www.ncbi.nlm.nih.gov/bioproject/PRJNA421373.(2018-10-1).

105. Fan R, Gu Z, Guang X, Marín JC, Varas V, Gonzalez B, Wheeler J, Hu Y, Li E, Sun X, Yang X, Zhang C, Gao W, He J, Munch K, Corbett-Detig R, Barbato M, Pan S, Zhan X, Bruford W M, Dong C. Lama guanicoe cacsilensis isolate: CD2-2017 Genome sequencing and assembly. NCBI Bioproject. 2018. https:/www.ncbi.nIm.nih.gov/bioproject/PRJNA427644.(2018-10-1).

106. Fan R, Gu Z, Guang X, Marín JC, Varas V, Gonzalez B, Wheeler J, Hu Y, Li E, Sun X, Yang X, Zhang C, Gao W, He J, Munch K, Corbett-Detig R, Barbato M, Pan S, Zhan X, Bruford W M, Dong C. Genomic analysis of the domestication and postSpanish conquest evolution of the llama and alpaca. NCBI Bioproject. 2020. https://www.ncbi.n/m.nih.gov/bioproject/ PRJNA612032.(2020-2-20).

107. Fan R, Gu Z, Guang X, Marín JC, Varas V, Gonzalez B, Wheeler J, Hu Y, Li E, Sun X, Yang X, Zhang C, Gao W, He J, Munch K, Corbett-Detig R, Barbato M, Pan S, Zhan X, Bruford W M, Dong C. Alpaca skin sequencing. NCBI Bioproject. 2020. https://www.ncbi.nlm.nih.gov/bioproject/PRJNA636766.(2020-6-2).

\section{Publisher's Note}

Springer Nature remains neutral with regard to jurisdictional claims in published maps and institutional affiliations.

Ready to submit your research? Choose BMC and benefit from:
- fast, convenient online submission
- thorough peer review by experienced researchers in your field
- rapid publication on acceptance
- support for research data, including large and complex data types
- gold Open Access which fosters wider collaboration and increased citations
- maximum visibility for your research: over 100M website views per year
At BMC, research is always in progress.
Learn more biomedcentral.com/submissions

